\title{
ROBUST STABILITY OF INPUT-OUTPUT SYSTEMS WITH INITIAL CONDITIONS*
}

\author{
JING LIU $^{\dagger}$ AND MARK FRENCH ${ }^{\dagger}$
}

\begin{abstract}
We consider the development of a general nonlinear input-output theory which encompasses systems with initial conditions. Systems are defined in a set theoretic manner from input-output pairs on a doubly infinite time axis, and a general construction of the initial conditions is given in terms of an equivalence class of trajectories on the negative time axis. Input-output operators are then defined for given initial conditions, and a suitable notion of input-output stability on the positive time axis with initial conditions is given. This notion of stability is closely related to the ISS/IOS concepts of Sontag. A fundamental robust stability theorem is derived which represents a generalization of the input-output operator robust stability theorem of Georgiou and Smith, to include the case of initial conditions. This includes a suitable generalization of the nonlinear gap metric. Some applications are given to show the utility of the robust stability theorem.
\end{abstract}

Key words. nonlinear systems, robust stability, gap metric, feedback connection, small-gain-like stability theorem, ISS/IOS

AMS subject classifications. 93C10, 93D09, 93D25, 93D15, 93D20

DOI. $10.1137 / 120903373$

1. Introduction. The general nonlinear input-output theory of systems was initiated in the 1960s by Zames [45, 46, 47] and Sandberg [25, 26]. It views systems as black boxes identified with operators mapping inputs to outputs. Such approaches, including recent contributions such as [13], do not include a systematic treatment of initial conditions. On the other hand, states and initial conditions have been introduced into input-output reasoning via the well-known input-to-state stability (ISS) theory due to Sontag [27] (and its many variants; see, e.g., [29, 30, 34]). The ISS approach is fundamentally a state space approach in which systems are assumed to have a known state space representation (and, e.g., Lyapunov constructions and characterizations play a significant role). In contrast, input/output approaches to robust stability are concerned with perturbations to nominal systems which induce significant (and potentially unknown) changes to the underlying state space. For example, while a nominal system may be modeled by a low order finite dimensional system, the true system is viewed as a perturbed system typically with a differing dimension, as occurs with a finite dimensional multiplicative perturbation, or represents a shift from a nominal model with a finite dimensional state space to an infinite dimensional system. For example, consider the nominal plant $\Sigma$ with one dimensional state space,

$$
\Sigma: \dot{x}(t)=\phi(x(t))+u(t), \quad y(t)=x(t),
$$

and the perturbed plant $\Sigma_{\tau}$ with infinite dimensional state space,

$$
\Sigma_{\tau}: \dot{x}(t)=\phi(x(t))+u(t-\tau), \quad y(t)=x(t), \quad 0<\tau \leq \tau_{0},
$$

where $\phi: \mathbb{R} \rightarrow \mathbb{R}$ is a memoryless nonlinear function satisfying a sector condition (Example 4.3). The plants $\Sigma$ and $\Sigma_{\tau}$ are close in the sense of nonlinear gap metric [13]

\footnotetext{
* Received by the editors December 21, 2012; accepted for publication (in revised form) January 20, 2015; published electronically June 25, 2015.

http://www.siam.org/journals/sicon/53-3/90337.html

†School of Electronics and Computer Science, University of Southampton, Southampton, SO17 1BJ, UK (j14g10@zepler.net, mcf@ecs.soton.ac.uk). Financial support for the first author was provided by CSC of China, BIS of UK, and ECS for a joint UK-China Scholarship for Excellence (SfE) over the last four years. 
$\left(\delta\left(\Sigma, \Sigma_{\tau}\right) \rightarrow 0\right.$ as $\left.\tau \rightarrow 0\right)$ but with different dimensional state spaces, and one would anticipate that a satisfactory feedback controller for $\Sigma$ will also work for $\Sigma_{\tau}$ for any $0<\tau \leq \tau_{0}$ provided $\tau_{0}$ is sufficiently small. The initial condition in $\Sigma$ can be taken to be $x(0) \in \mathbb{R}$. However, for $\Sigma_{\tau}$ the initial condition is necessarily infinite dimensional, e.g., $\left(x(0),\left.u\right|_{(-\tau, 0]}\right) \in \mathbb{R} \times L^{2}(-\tau, 0]$. Intuitively, even when initial conditions are taken into consideration, the nominal plant $\Sigma$ when stabilized by a controller should remain stabilized when replaced by any of the perturbed plants $\Sigma_{\tau}, 0<\tau \leq \tau_{0}$. Clearly, to quantify such statements, we need appropriate notions of stability together with an appropriate quantification of the notion of "size" of initial conditions which can be consistently applied across $\Sigma$ and $\Sigma_{\tau}$ for any $0<\tau \leq \tau_{0}$. Additionally, these concepts must also be applicable to all other "reasonable" perturbations (multiplicative, additive, etc.), which often change the state space structure and move the scope of the required framework beyond that of state space representations.

The purpose of this paper is to develop a general input/output framework which incorporates a general concept of initial conditions. The central result obtained is a generalization of the robust stability results of [13], whereby the initial conditions (characterized by a purely input-output formalism drawn from [41]) are reflected within the stability concept in an ISS-like manner (cf. [27, 29, 30, 34]). We remark that a central assumption in [13] is a requirement that systems are defined on semiinfinite time axes and map zero inputs onto zero outputs. Implicitly, this requires that the systems have zero initial conditions. There are a number of later extensions which permit consideration of nonzero responses to zero disturbances, e.g., [8, 12], however, neither of these approaches are directly aimed at the case of initial conditions and cannot directly be used to establish fading memory properties. Explicit robust stability results are given in $[7,9]$ for a specific case of a linear plant and a nonlinear controller with initial conditions. A more general construction for nonlinear plants can be found in [10, section 7], and this forms the basis for this contribution.

Thus, on the one hand, the contribution of this paper can be viewed as a generalization of the ISS approach to enable a realistic treatment of robust stability in the context of perturbations which fundamentally change the structure of the state space, and on the other hand, can be viewed as a generalization of existing operator-based input-output approaches to robust stability to include initial conditions within, in particular, the nonlinear gap formalism of [13].

This paper is organized as follows. In section 2, we introduce definitions of systems, initial conditions, and closed-loop systems, which involve only input-output structures. In section 2 we show how the general initial condition construction relates to standard notions of initial conditions for systems having particular representations. The fundamental robust stability theorem for input-output feedback systems with initial conditions in terms of a generalized gap metric is given in section 3. Section 4 considers some applications to show the effects of this paper's results. We draw conclusions in section 5 .

2. Systems, initial conditions and closed-loop systems. Let $\mathcal{S}$ denote the set of all locally integrable maps $\mathbb{R} \rightarrow \mathcal{X}$ where $\mathcal{X}$ is a nonempty set. For any interval $J$, we regard $\mathcal{S}_{J}$ as a subspace of $\mathcal{S}$ by identifying $\mathcal{S}_{J}$ with the set of maps in $\mathcal{S}$ which vanish outside of $J$. We define a truncation operator $T_{J}: \mathcal{S} \rightarrow \mathcal{S}$ and a restriction operator $R_{J}: \mathcal{S}_{I} \rightarrow \mathcal{S}_{J}$ with $J \subseteq I$ as follows:

$$
\begin{aligned}
& T_{J}: \mathcal{S} \rightarrow \mathcal{S}, v \mapsto T_{J} v \triangleq\left(t \mapsto\left\{\begin{array}{cc}
v(t), & t \in J \\
0 & \text { otherwise }
\end{array}\right) ;\right. \\
& R_{J}: \mathcal{S}_{I} \rightarrow \mathcal{S}_{J}, \quad v \mapsto R_{J} v \triangleq(t \mapsto v(t), \quad t \in J) .
\end{aligned}
$$

Copyright $@$ b by SIAM. Unauthorized reproduction of this article is prohibited. 
We let $R_{+} \triangleq R_{[0, \infty)}$ and $R_{-} \triangleq R_{(-\infty, 0]}$. For any $u, v \in \mathcal{S}$ and any $\tau \in \mathbb{R}$, the $\tau$-concatenation of $u$ and $v$, denoted $u \wedge_{\tau} v$, is defined by $\left(u \wedge_{\tau} v\right)(t)=u(t)$ if $t<\tau$ and $\left(u \wedge_{\tau} v\right)(t)=v(t)$ if $t \geq \tau$. We abbreviate $u \wedge v \triangleq u \wedge_{0} v$. Define $\mathcal{V} \subseteq \mathcal{S}$ to be a signal space if and only if it is a vector space. Suppose additionally that $\mathcal{V}$ is a normed vector space and the norm $\|\cdot\|=\|\cdot\|_{\mathcal{V}}$ is also defined for signals of the form $T_{J} v, v \in \mathcal{V}, J \subseteq \mathbb{R}$. We define a norm $\|\cdot\|_{J}$ on $\mathcal{S}_{J}$ by $\|v\|_{J}=\left\|T_{J} v\right\|$ for $v \in \mathcal{S}_{J}$ (define $\|v\|_{J} \triangleq \infty$ if $\left.T_{J} v \in \mathcal{S} \backslash \mathcal{V}\right)$. The extended space $\mathcal{V}_{e}$ of $\mathcal{V}$ is defined by

$$
\mathcal{V}_{e} \triangleq\left\{v \in \mathcal{S} \mid \forall a, b,(-\infty<a<b<\infty): T_{(a, b)} v \in \mathcal{V}\right\}
$$

and the interval space $\mathcal{V}(J) \triangleq R_{J} \mathcal{V}$ for any $J \subseteq \mathbb{R}$; we also abbreviate $\mathcal{V}^{+}=R_{+} \mathcal{V}$, $\mathcal{V}^{-}=R_{-} \mathcal{V}, \mathcal{V}_{e}^{+}=R_{+} \mathcal{V}_{e}$, and $\mathcal{V}_{e}^{-}=R_{-} \mathcal{V}_{e}$. In the rest of this paper, unless specified otherwise, we always let $\mathcal{U}, \mathcal{Y}$ be normed (input/output) signal spaces (such as $\left.L^{q}\left(\mathbb{R} ; \mathbb{R}^{n}\right), 1 \leq q \leq \infty\right)$ with norm $\|\cdot\|_{\mathcal{U}},\|\cdot\|_{\mathcal{Y}}$, respectively. Let $\mathcal{W} \triangleq \mathcal{U} \times \mathcal{Y}$ with the product norm defined in the usual way, $\|(u, y)\|_{\mathcal{W}}=\left(\|u\|_{\mathcal{U}}^{q}+\|y\|_{\mathcal{Y}}^{q}\right)^{\frac{1}{q}}$ if $q \geq 1$ and $\|(u, y)\|_{\mathcal{W}}=\max \left\{\|u\|_{\mathcal{U}},\|y\|_{\mathcal{Y}}\right\}$ if $q=\infty$.

2.1. Systems. In an input/output framework, it is only the relationship between inputs and outputs that is a priori relevant. In this sense, notions of "system" and of "stability" should be made without the axiomatical postulation of state.

Definition 2.1. Given normed signal spaces $\mathcal{U}, \mathcal{Y}$ and $\mathcal{W} \triangleq \mathcal{U} \times \mathcal{Y}$, a system $Q$ is defined via the specification of a subset $\mathfrak{B}_{Q} \subseteq \mathcal{W}_{e}$.

The signal pair $(u, y) \in \mathcal{U}_{e} \times \mathcal{Y}_{e}$ is called an input-output pair. At this stage, we do not impose any further requirements on the input/output partition. In an operator-based input/output framework (e.g., [13]), it would be typical to start with an operator $Q: \mathcal{U}_{e}^{+} \rightarrow \mathcal{Y}_{e}^{+}$, where, e.g., $\mathcal{U}_{e}^{+}=\mathcal{Y}_{e}^{+} \triangleq L_{e}^{2}\left(\mathbb{R}_{+} ; \mathbb{R}\right)$ and $Q(0)=0$. Note that the above definition of a system differs from both Zames's representation of inputoutput systems by operators [44] and Willems's structure of input-output systems by behaviors with input/output partition [23]. Here, we allow both $\left(u, y_{1}\right)$ and $\left(u, y_{2}\right)$ with $y_{1} \neq y_{2}$ to belong to the same set $\mathfrak{B}_{Q}$. And it does not require that for any $u \in \mathcal{U}_{e}$ there exists a $y \in \mathcal{Y}_{e}$ such that $(u, y) \in \mathfrak{B}_{Q}$. For example, Let $\mathcal{U}=\mathcal{Y} \triangleq L^{2}(\mathbb{R} ; \mathbb{R})$ and consider the system $Q$ represented by the set $\mathfrak{B}_{Q}=\left\{(u, y) \in \mathcal{U}_{e} \times \mathcal{Y}_{e} \mid y^{2}=u\right\}$. It is easy to verify that for $u(t)=e^{-2|t|}, t \in \mathbb{R}$ and $y(t)=e^{-|t|}, t \in \mathbb{R}$, we have both $(u, y)$ and $(u,-y)$ belonging to $\mathfrak{B}_{Q}$, and that for $u(t)=-e^{-2|t|}, t \in \mathbb{R}$, there is no $y \in L_{e}^{2}(\mathbb{R} ; \mathbb{R})$ such that $(u, y) \in \mathfrak{B}_{Q}$. Since our set $\mathfrak{B}_{Q}$ allows us to consider multivalued maps $Q$ or relations $Q$, we will see in subsequent sections that this is key to our unified treatment of initial conditions.

Definition 2.2. A system $Q$ is said to be linear if the set $\mathfrak{B}_{Q}$ is a vector space, i.e., $\lambda_{1} w_{1}+\lambda_{2} w_{2} \in \mathfrak{B}_{Q}$ for any $w_{1}, w_{2} \in \mathfrak{B}_{Q}$ and any $\lambda_{1}, \lambda_{2} \in \mathbb{R}$. It is said to be time-invariant if $w \in \mathfrak{B}_{Q}$ implies $w(\cdot+\tau) \in \mathfrak{B}_{Q} \forall \tau \in \mathbb{R}$.

Definition 2.3. Given normed signal spaces $\mathcal{U}$ and $\mathcal{Y}$, an operator $\Phi: \mathcal{U}_{e}^{+} \rightarrow \mathcal{Y}_{e}^{+}$ is said to be causal if

$$
\forall u, v \in \mathcal{U}_{e}^{+}, \forall t>0:\left[\left.u\right|_{[0, t]}=\left.\left.v\right|_{[0, t]} \Rightarrow(\Phi u)\right|_{[0, t]}=\left.(\Phi v)\right|_{[0, t]}\right],
$$

while a system $Q$ is said to be causal if

$$
\forall\left(u, y_{u}\right),\left(v, y_{v}\right) \in \mathfrak{B}_{Q}, \forall t \in \mathbb{R}:\left[\left.u\right|_{(-\infty, t]}=\left.\left.v\right|_{(-\infty, t]} \Rightarrow \mathfrak{B}_{Q}^{u}\right|_{(-\infty, t]}=\left.\mathfrak{B}_{Q}^{v}\right|_{(-\infty, t]}\right],
$$

where $\mathfrak{B}_{Q}^{u} \triangleq\left\{w \in \mathcal{W}_{e} \mid \exists y \in \mathcal{Y}_{e}\right.$ such that $\left.w=(u, y) \in \mathfrak{B}_{Q}\right\}$.

This definition generalizes the definition of a casual operator. Note that any operator $\Phi: \mathcal{U}_{e}^{+} \rightarrow \mathcal{Y}_{e}^{+}$can be represented by a system $\mathfrak{B}_{\Phi}=\left\{(u, y) \in \mathcal{U}_{e} \times \mathcal{Y}_{e} \mid R_{-} y=\right.$ 
$\left.R_{-} u=0, R_{+} y=\Phi\left(R_{+} u\right)\right\}$. According to above definition, the operator $\Phi$ is causal if and only if the system $\mathfrak{B}_{\Phi}$ is causal. We will be interested to define system properties using trajectories defined on the positive half-line $[t, \infty)$. In order to define the well-posedness of a system, we first introduce the two properties of existence and uniqueness of a system. In the following, we fixed initial time $t=0$ if not otherwise specified and use the notation $\mathfrak{B}_{Q}^{-}$defined as follows to denote the system $Q$ 's past trajectories:

$$
\mathfrak{B}_{Q}^{-} \triangleq R_{-} \mathfrak{B}_{Q}=\left\{w_{-} \in \mathcal{W}_{e}^{-} \mid \exists w_{+} \in \mathcal{W}_{e}^{+} \text {, s.t. } w_{-} \wedge w_{+} \in \mathfrak{B}_{Q}\right\} .
$$

Definition 2.4. A system $Q$ is said to have the existence property if for any $w_{-} \in \mathfrak{B}_{Q}^{-}$and any $u_{+} \in \mathcal{U}_{e}^{+}$there exists a $y_{+} \in \mathcal{Y}_{e}^{+}$such that $w_{-} \wedge\left(u_{+}, y_{+}\right) \in \mathfrak{B}_{Q} ;$ it is said to have the uniqueness property if for any $w_{-} \in \mathfrak{B}_{Q}^{-}$and any $u_{+} \in \mathcal{U}_{e}^{+}$,

$$
w_{-} \wedge\left(u_{+}, y_{+}\right), w_{-} \wedge\left(u_{+}, \tilde{y}_{+}\right) \in \mathfrak{B}_{Q} \text { with } y_{+}, \tilde{y}_{+} \in \mathcal{Y}_{e}^{+} \Rightarrow y_{+}=\tilde{y}_{+} ;
$$

and it is well-posed if it has both the existence and uniqueness properties.

Well-posedness means that future output $y_{+}$can be deduced from the set $\mathfrak{B}_{Q}$ (representing system properties), the past input-output pair $\left(u_{-}, y_{-}\right)$, and the future input $u_{+}$. The uniqueness property is equivalent to the concept that output processes input as defined in [42]. The graph $\mathcal{G}_{Q}^{w_{-}}$of a system $Q$ for a given past trajectory $w_{-} \in \mathfrak{B}_{Q}^{-}$is defined by

$$
\mathcal{G}_{Q}^{w_{-}} \triangleq\left\{w_{+} \in \mathcal{W}^{+} \mid w_{-} \wedge w_{+} \in \mathfrak{B}_{Q}\right\} \subseteq \mathcal{W}^{+} .
$$

We conclude this section with the following results, which will be used in the proof of Theorem 3.2 below.

Proposition 2.5. For any system $Q$, suppose that $Q$ is causal and has the uniqueness property. Then for any $w_{-} \triangleq\left(u_{-}, y_{-}\right) \in \mathfrak{B}_{Q}^{-}$, any $w_{+} \triangleq\left(u_{+}, y_{+}\right) \in \mathcal{W}_{e}^{+}$, any $\tilde{w}_{+} \triangleq\left(\tilde{u}_{+}, \tilde{y}_{+}\right) \in \mathcal{W}_{e}^{+}$, and any $\tau \in(0, \infty)$, we have

$$
w_{-} \wedge w_{+}, w_{-} \wedge \tilde{w}_{+} \in \mathfrak{B}_{Q} \text { with }\left.u_{+}\right|_{[0, \tau)}=\left.\left.\tilde{u}_{+}\right|_{[0, \tau)} \Rightarrow y_{+}\right|_{[0, \tau)}=\left.\tilde{y}_{+}\right|_{[0, \tau)} .
$$

Proof. Define $w \triangleq(u, y) \triangleq\left(u_{-} \wedge u_{+}, y_{-} \wedge y_{+}\right)$and $\tilde{w} \triangleq(\tilde{u}, \tilde{y}) \triangleq\left(u_{-} \wedge \tilde{u}_{+}, y_{-} \wedge \tilde{y}_{+}\right)$. It's easy to verify that $w=w_{-} \wedge w_{+}$and $\tilde{w}=w_{-} \wedge \tilde{w}_{+}$. Since the system $Q$ is causal and $\left.u\right|_{(-\infty, \tau)}=\left.\tilde{u}\right|_{(-\infty, \tau)}$ (note that $\left.\left.u_{+}\right|_{[0, \tau)}=\left.\tilde{u}_{+}\right|_{[0, \tau)}\right)$, we obtain that $\left.\mathfrak{B}_{Q}^{u}\right|_{(-\infty, \tau)}=$ $\left.\mathfrak{B}_{Q}^{\tilde{u}}\right|_{(-\infty, \tau)}$ with $\mathfrak{B}_{Q}^{u}$ defined as in Definition 2.3. It follows from the fact $\left.(u, y)\right|_{(-\infty, \tau)}$ $\left.\in \mathfrak{B}_{Q}^{u}\right|_{(-\infty, \tau)}=\left.\mathfrak{B}_{Q}^{\tilde{u}}\right|_{(-\infty, \tau)}$ that there exists a $\hat{y} \triangleq \hat{y}_{-} \wedge \hat{y}_{+} \in \mathcal{Y}_{e}$ satisfying $(\tilde{u}, \hat{y}) \in$ $\mathfrak{B}_{Q}^{\tilde{u}} \subseteq \mathcal{W}_{e}$ and $\left.(\tilde{u}, \hat{y})\right|_{(-\infty, \tau)}=\left.(u, y)\right|_{(-\infty, \tau)}=\left.w\right|_{(-\infty, \tau)}$. Hence, we have $\hat{y}_{-}=y_{-}$and $\left.\hat{y}_{+}\right|_{[0, \tau)}=\left.y_{+}\right|_{[0, \tau)}$. To conclude the proof, we only have to show that $\left.\hat{y}_{+}\right|_{[0, \tau)}=\left.\tilde{y}_{+}\right|_{[0, \tau)}$. This follows directly from the uniqueness property of the system $Q$ and the fact that $w_{-} \wedge\left(\tilde{u}_{+}, \tilde{y}_{+}\right)=\tilde{w} \in \mathfrak{B}_{Q}$ and $w_{-} \wedge\left(\tilde{u}_{+}, \hat{y}_{+}\right)=(\tilde{u}, \hat{y}) \in \mathfrak{B}_{Q}^{\tilde{u}} \subseteq \mathfrak{B}_{Q}$. (In fact, we have $\left.\hat{y}_{+}=\tilde{y}_{+} \cdot\right)$

Corollary 2.6. For any system $Q$, suppose that $Q$ is causal and has the uniqueness property. If for any $w_{-} \in \mathfrak{B}_{Q}^{-}$, any $u_{+} \in \mathcal{U}_{e}^{+}$, and any $\tau \in(0, \infty)$, there exists $a y_{+}^{\tau} \in \mathcal{Y}_{e}^{+}$such that $\left.\left.\left[w_{-} \wedge\left(u_{+}, y_{+}^{\tau}\right)\right]\right|_{(-\infty, \tau)} \in \mathfrak{B}_{Q}\right|_{(-\infty, \tau)}$, then the system $Q$ is wellposed.

Proof. We only need to show that the system $Q$ has the existence property. To this end, fix any $w_{-} \in \mathfrak{B}_{Q}^{-}$and any $u_{+} \in \mathcal{U}_{e}^{+}$and define a time function $y_{+}(t)$ on the positive infinite interval, $0 \leq t<\infty$, as follows: for any $t \geq 0$, choose some $\tau \in(0, \infty)$ 
with $\tau>t$, and let $y_{+}(t) \triangleq y_{+}^{\tau}(t)$. This function $y_{+}$is well-defined. ${ }^{1}$ It follows from the definition of $\mathcal{Y}_{e}^{+}$that $y_{+} \in \mathcal{Y}_{e}^{+}$, since $\left.y_{+}\right|_{[0, \tau)}=\left.y_{+}^{\tau}\right|_{[0, \tau)}$ with $y_{+}^{\tau} \in \mathcal{Y}_{e}^{+} \forall 0<\tau<\infty$. To conclude the proof, we need to show $w_{-} \wedge\left(u_{+}, y_{+}\right) \in \mathfrak{B}_{Q}$. This is obvious since $\left.\left[w_{-} \wedge\left(u_{+}, y_{+}\right)\right]\right|_{(-\infty, \tau)}=\left.\left.\left[w_{-} \wedge\left(u_{+}, y_{+}^{\tau}\right)\right]\right|_{(-\infty, \tau)} \in \mathfrak{B}_{Q}\right|_{(-\infty, \tau)} \forall 0<\tau<\infty$.

2.2. Initial conditions. As discussed in intuitive terms in the control literature, see [43, Chapter 1] and [45], the state is a classifier of input-output pasts and the state should contain all the information of past history of the system which at any time together with the future input completely determine the future output. The state at time 0 thus determines the initial conditions. In the following, we will give a precise way to define the state of an arbitrary input/output system. It is fundamental that the construction does not require a system representation, but we do show how the construction relates to the standard concepts of state for significant classes of system representations. The genesis of this approach lies in [10, section 7]. From the viewpoint of observability, for any observable nonlinear system represented by a state space model, the initial state can be reconstructed from observed output signals given some known input signals (see, e.g., [11]).

We now define an equivalence relation on $\mathfrak{B}_{Q}^{-} \triangleq R_{-} \mathfrak{B}_{Q}$ (see (2.1)) as follows: for any $w_{-}, \tilde{w}_{-} \in \mathfrak{B}_{Q}^{-}$, we say

$$
w_{-} \sim \tilde{w}_{-} \Leftrightarrow Q^{w_{-}}\left(u_{+}\right)=Q^{\tilde{w}_{-}}\left(u_{+}\right) \forall u_{+} \in \mathcal{U}_{e}^{+},
$$

where $Q^{w_{-}}\left(u_{+}\right)$denotes the set (possibly empty) of all future outputs generated by the system past input-output $w_{-} \in \mathfrak{B}_{Q}^{-}$and future input $u_{+} \in \mathcal{U}_{e}^{+}$, i.e.,

$$
Q^{w_{-}}\left(u_{+}\right) \triangleq\left\{y_{+} \in \mathcal{Y}_{e}^{+} \mid w_{-} \wedge\left(u_{+}, y_{+}\right) \in \mathfrak{B}_{Q}\right\} .
$$

The equivalence class of any $w_{-}$in $\mathfrak{B}_{Q}^{-}$is denoted by $\left[w_{-}\right] \triangleq\left\{\tilde{w}_{-} \in \mathfrak{B}_{Q}^{-} \mid \tilde{w}_{-} \sim w_{-}\right\}$.

Definition 2.7. We define $\mathfrak{S}_{Q}$ the initial state space of $Q$ at initial time 0 as the quotient set $\mathfrak{B}_{Q}^{-} / \sim$, i.e., $\mathfrak{S}_{Q}=\mathfrak{B}_{Q}^{-} / \sim \triangleq\left\{\left[w_{-}\right] \mid w_{-} \in \mathfrak{B}_{Q}^{-}\right\}$.

From the equivalence relation $\sim$, for any $x_{0} \in \mathfrak{S}_{Q}$ we define the set $Q^{x_{0}}\left(u_{+}\right)$by

$$
Q^{x_{0}}\left(u_{+}\right) \triangleq Q^{w_{-}}\left(u_{+}\right) \quad \forall u_{+} \in \mathcal{U}_{e}^{+}, \forall w_{-} \in x_{0}
$$

If the initial time is chosen to be $t_{0} \in \mathbb{R}$, we can similarly define the initial state space denoted by $\mathfrak{S}_{Q}^{t_{0}}$ of a system $Q$ at initial time $t_{0}$ by the same procedure. Note that the above definition of initial state space doesn't require the system to be well-posed; however, if so, then there is a unique element in $Q^{w_{-}}\left(u_{+}\right)$for every $w_{-} \in \mathfrak{B}_{Q}^{-}$and every $u_{+} \in \mathcal{U}_{e}^{+}$; and in this case, $Q^{w_{-}(\cdot)}$ can be regarded as an operator from $\mathcal{U}_{e}^{+}$to $\mathcal{Y}_{e}^{+}$for every $w_{-} \in \mathfrak{B}_{Q}^{-}$. In turn, this implies that for every $x_{0} \in \mathfrak{S}_{Q}, Q^{x_{0}}(\cdot)$ is an operator from $\mathcal{U}_{e}^{+}$to $\mathcal{Y}_{e}^{+}$.

This equivalence class construction of the initial state space is not new; it is closely related to the construction of states in automata (or machine) theory and control theory via Nerode equivalence appearing in a slightly different manner. This technique was introduced by Nerode [22] when defining a state-equivalence relation in linear automata theory. The formal definition of Nerode equivalence can be found in [24, p. 114] in the general setting of automata theory including the nonlinear case; in $[18,3]$ for discrete-time systems from an abstract algebraic point of view; in [19, Chapters 7 and

\footnotetext{
${ }^{1}$ To see this, it suffices to show that $\left.y_{+}^{\tau_{1}}\right|_{\left[0, \tau_{1}\right)}=\left.y_{+}^{\tau_{2}}\right|_{\left[0, \tau_{1}\right)}$ for any $0<\tau_{1}<\tau_{2}<\infty$. This follows directly from Proposition 2.5, since the system $Q$ is causal and has the uniqueness property.
} 
10] including a discussion of connection between automata and control theory; and in [32, p. 309] for any time-invariant input/output behaviors including both discrete-time and continuous-time cases. A concrete approach to the Nerode equivalence construction for discrete time transfer functions was studied in [18, pp. 315 and 470], as well as for continuous time transfer functions in [21]. The equivalence relation considered in this paper is slightly different from the one considered in standard texts (see, e.g., [32, p. 309]), where equivalence classes only relate to input sequences, since we do not restrict ourself to input/output behaviors which can be associated with an input/output map, and hence the equivalence class is constructed from both input and output pairs.

Within the behavioral approach, Willems constructs three canonical state representations by introducing three equivalence relations for a given system represented by a behavior [41]. The construction of state in this paper is similar to the past-induced canonical state representation in [41]. Note that in this paper, we do not impose any requirements on the input/output partition for a system (see Definition 2.1). This construction of state enables us to define the well-posedness of a system and a closedloop system in a unified way (see below). Notice that this is different from giving a definition of well-posedness for a system with Willems's input/output partition [23, Definition 3.3.1], since any systems with Willems's input/output partition already guarantee the existence property, which is a very important property of a closed-loop system. Hence, we relax the requirement that the input is free in [23, Definition 3.3.1] in order to study closed-loop systems.

A functional $\chi$ assigns a notion of size to elements in the initial state space $\mathfrak{S}_{Q}$ :

$$
\chi: \quad \mathfrak{S}_{Q} \rightarrow[0, \infty], \quad x_{0} \mapsto \chi\left(x_{0}\right) \triangleq \inf \left\{\left\|w_{-}\right\| \mid w_{-} \in x_{0}\right\} .
$$

This notion of size defined above related to finite energy reachability may be interpreted as the minimization of energy of the past system trajectories that "explain" the corresponding initial state. Notice that in section 3.2, we will give a detailed discussion about the concept of finite-time reachability, which roughly means that any state can be reached from zero state by finite time. The notion of size defined above may also be interpreted as the required supply in the context of dissipative dynamical systems; see, e.g., $[39,40]$. The determination of $\chi$ is a standard problem in optimal control; see, e.g., [1]. It is well known that, for $L^{2}$ norm (square-integrable) in the linear case $\dot{x}=A x+B u, y=C x+D u$, the above infimum is simplified to the traditional linear quadratic optimal control problem with $\left\|w_{-}\right\|^{1 / 2}=\int_{-\infty}^{0}\left[u^{T}(t) u(t)+y^{T}(t) y(t)\right] d t$. Moreover, if the considered linear system is minimal (i.e., $(A, B)$ controllable and $(A, C)$ observable) and $D+D^{T}$ is invertible, then there exists a real symmetric nonnegative definite matrix $K$ such that $\chi\left(x_{0}\right)=\left(s_{0}^{T} K s_{0}\right)^{1 / 2}$; see, e.g., [38], where $s_{0} \in \mathbb{R}^{n}$ is one-to-one related to $x_{0} \in \mathfrak{S}_{Q}$ by the bijection obtained from Corollary 2.12.

We conclude this section by giving some properties of $\mathfrak{S}_{Q}$ and $\chi$ for the linear systems.

Proposition 2.8. If the system $Q$ is linear, then the initial state space $\mathfrak{S}_{Q}$ is a vector space. Moreover, the functional $\chi$ given by (2.6) defines a norm on $\mathfrak{S}_{Q}$.

Proof. It is elementary to show that the initial state space $\mathfrak{S}_{Q}$ is a vector space with $\mathbf{0}=\left[\left.0\right|_{(-\infty, 0]}\right]$ as its additive identity and satisfies $\chi(\mathbf{0})=0$. From the definition of $\chi$ (see (2.6)), it is easy to see that $\chi\left(z_{0}\right) \geq 0$ for any $z_{0} \in \mathfrak{S}_{Q}$ and that if $\chi\left(z_{0}\right)=0$, then we must have $\left.0\right|_{(-\infty, 0]} \in z_{0}$ (i.e., $\left.z_{0}=\mathbf{0}\right)$. For any $x_{0}=\left[w_{-}\right] \in \mathfrak{S}_{Q}$ and any $\lambda \in$ $\mathbb{R}$, we have $\chi\left(\lambda \cdot x_{0}\right)=\chi\left(\left[\lambda \cdot w_{-}\right]\right)=|\lambda| \chi\left(\left[w_{-}\right]\right)=|\lambda| \chi\left(x_{0}\right)$. For any $w_{1_{-}}, w_{2_{-}} \in \mathfrak{B}_{Q}^{-}$ we have $\left\|w_{1-}+w_{2-}\right\| \leq\left\|w_{1-}\right\|+\left\|w_{2-}\right\|$, and thus we obtain $\chi\left(x_{0}+y_{0}\right) \leq \chi\left(x_{0}\right)+\chi\left(y_{0}\right)$ for any $x_{0}, y_{0} \in \mathfrak{S}_{Q}$. Therefore, $\chi$ defines a norm on $\mathfrak{S}_{Q}$ for any linear system $Q$. 
2.2.1. The relation to state space initial conditions. Consider a system $\Sigma$ described by the state-space model

$$
\dot{x}=f(x, u), \quad y=h(x, u),
$$

where $u(t) \in \mathbb{R}^{m}(t \in \mathbb{R})$ is the input variable, $x(t) \in M \subseteq \mathbb{R}^{l}$ denotes the state variable ( $M$ is an open set), $y(t) \in \mathbb{R}^{p}$ represents the output variable, and both $f: M \times \mathbb{R}^{m} \rightarrow M$ and $g: M \times \mathbb{R}^{m} \rightarrow \mathbb{R}^{p}$ are continuous functions. Define signal spaces $\mathcal{U} \triangleq L^{q}\left(\mathbb{R} ; \mathbb{R}^{m}\right)(1 \leq q \leq \infty), \mathcal{Y} \triangleq L^{q}\left(\mathbb{R} ; \mathbb{R}^{p}\right)(1 \leq q \leq \infty)$, and $\mathcal{W} \triangleq \mathcal{U} \times \mathcal{Y}$. According to Definition 2.1, the system $\Sigma$ is defined by the set

$$
\mathfrak{B}_{\Sigma}=\left\{w \in \mathcal{W}_{e} \mid w=(u, y) \text { and (2.7) satisfies for some } x(t) \in M(t \in \mathbb{R})\right\}
$$

By using the same procedure in section 2.2 , we can define the initial state space $\mathfrak{S}_{\Sigma}$ for the above set $\mathfrak{B}_{\Sigma}$ at initial time 0 .

Definition 2.9. The state space model (2.7) is said to be forward complete [2] if for any $u_{+} \in \mathcal{U}_{e}^{+}$and any initial state $x_{0} \in M$, there exists a unique $x(t) \in M$ $(\forall t \geq 0)$ satisfying (2.7). It is said to be backward complete if for every $u_{-} \in \mathcal{U}_{e}^{-}$ and every initial state $x_{0}$, there exists a unique $x(t) \in M(\forall t \leq 0)$ satisfying (2.7). It is said to be complete if it is both forward complete and backward complete.

It is well known that the state space model (2.7) is complete if $f$ is continuous in $t$ and $u$ and Lipschitz continuous in $x$ (see, e.g., [4]). Suppose that the state space model (2.7) is a complete representation. If the trajectories of (2.7) are required to satisfy the initial condition $x(0)=x_{0}\left(x_{0} \in M\right)$, then the state space model defines a forward operator $\Sigma_{+}^{x_{0}}$ from $\mathcal{U}_{e}^{+}$to $\mathcal{Y}_{e}^{+}$as follows: each input $u_{+} \in \mathcal{U}_{e}^{+}$gives rise to a solution $x(t) \in M(t \geq 0)$ of $\dot{x}=f(x, u)$ satisfying the initial condition $x(0)=x_{0}$. This in turn defines an output $y_{+} \in \mathcal{Y}_{e}^{+}$by $y_{+}(t)=h\left(x(t), u_{+}(t)\right)(t \geq 0)$, i.e.,

$$
\Sigma_{+}^{x_{0}}: \mathcal{U}_{e}^{+} \rightarrow \mathcal{Y}_{e}^{+}, \quad u_{+} \mapsto y_{+} .
$$

A backward operator $\Sigma_{-}^{x_{0}}: \mathcal{U}_{e}^{-} \rightarrow \mathcal{Y}_{e}^{-}$can be similarly defined like (2.9).

Definition 2.10. Suppose that the state space model (2.7) is complete. It is said to be forward observable if (see, e.g., [14]), for any initial states $x_{0}, x_{0}^{\prime} \in M$ with $x_{0} \neq x_{0}^{\prime}$, there exists some $u_{+} \in \mathcal{U}_{e}^{+}$such that $\Sigma_{+}^{x_{0}}\left(u_{+}\right) \neq \Sigma_{+}^{x_{0}^{\prime}}\left(u_{+}\right)$. It is said to be strongly forward observable if, for any initial states $x_{0}, x_{0}^{\prime} \in M$ with $x_{0} \neq x_{0}^{\prime}$, for any $u_{+} \in \mathcal{U}_{e}^{+}$, we have $\Sigma_{+}^{x_{0}}\left(u_{+}\right) \neq \Sigma_{+}^{x_{0}^{\prime}}\left(u_{+}\right)$. It is said to be backward observable if, for any initial states $x_{0}, x_{0}^{\prime} \in M$ with $x_{0} \neq x_{0}^{\prime}$, there exist some $u_{-} \in \mathcal{U}_{e}^{-}$such that $\Sigma_{-}^{x_{0}}\left(u_{-}\right) \neq \Sigma_{-}^{x_{0}^{\prime}}\left(u_{-}\right)$. It is said to be strongly backward observable if, for any initial states $x_{0}, x_{0}^{\prime} \in M$ with $x_{0} \neq x_{0}^{\prime}$, for any $u_{-} \in \mathcal{U}_{e}^{-}$, we have $\Sigma_{-}^{x_{0}}\left(u_{-}\right) \neq \Sigma_{-}^{x_{0}^{\prime}}\left(u_{-}\right)$.

We let $\mathfrak{B}_{\Sigma}^{-}\left(x_{0}\right)$ denote the set of all past input-output trajectories which are compatible with the initial state $x_{0} \in M$ at initial time 0 :

$$
\mathfrak{B}_{\Sigma}^{-}\left(x_{0}\right) \triangleq\left\{\left(\begin{array}{l}
u_{-} \\
y_{-}
\end{array}\right) \mid \begin{array}{c}
u_{-} \in \mathcal{U}_{e}^{-}, y_{-} \in \mathcal{Y}_{e}^{-} \text {and }(2.7) \text { satisfies } \\
\text { for some } x(t) \in M(t \leq 0) \text { with } x(0)=x_{0}
\end{array}\right\} .
$$

Proposition 2.11. Suppose that the state space model (2.7) is complete, forward observable, and strongly backward observable. Then $F: x_{0} \mapsto \mathfrak{B}_{\Sigma}^{-}\left(x_{0}\right)$ defines a bijection from $M$ to $\mathfrak{S}_{\Sigma}$. 
Proof. From Definition 2.7, the initial state space at time 0 of $\mathfrak{B}_{\Sigma}$ is defined by $\mathfrak{S}_{\Sigma} \triangleq \mathfrak{B}_{\Sigma}^{-} / \sim$ with $\mathfrak{B}_{\Sigma}^{-} \triangleq R_{-} \mathfrak{B}_{\Sigma}$ (see (2.1)), and the corresponding equivalence relation $\sim$ on $\mathfrak{B}_{\Sigma}^{-}$is defined as follows (see (2.4) and (2.3)): for any $w_{-}, \tilde{w}_{-} \in \mathfrak{B}_{\Sigma}^{-}$,

$$
w_{-} \sim \tilde{w}_{-} \Leftrightarrow \Sigma^{w_{-}}\left(u_{+}\right)=\Sigma^{\tilde{w}_{-}}\left(u_{+}\right), \forall u_{+} \in \mathcal{U}_{e}^{+} .
$$

We obtain from (2.8) and (2.10) that $\mathfrak{B}_{\Sigma}^{-}=\bigcup_{x_{0} \in M}\left\{\mathfrak{B}_{\Sigma}^{-}\left(x_{0}\right)\right\}$. Since the state space model (2.7) is complete and strongly backward observable, we have $\mathfrak{B}_{\Sigma}^{-}\left(x_{0}\right) \cap$ $\mathfrak{B}_{\Sigma}^{-}\left(x_{0}^{\prime}\right)=\emptyset$ for any $x_{0}, x_{0}^{\prime} \in M$ with $x_{0} \neq x_{0}^{\prime}$. In addition, for any $w_{-} \in \mathfrak{B}_{\Sigma}^{-}\left(x_{0}\right)$ and any $u_{+} \in \mathcal{U}_{e}^{+}$, we have $\Sigma^{w_{-}}\left(u_{+}\right)=\Sigma_{+}^{x_{0}}\left(u_{+}\right)$with $\Sigma_{+}^{x_{0}}\left(u_{+}\right)$defined by $(2.9)$. Thus, for any $x_{0} \in M$, the set $\mathfrak{B}_{\Sigma}^{-}\left(x_{0}\right)$ is a subset of some equivalence class related to the equivalence relation $\sim$. Since the state space model (2.7) is also forward observable, (i.e., $\Sigma_{+}^{x_{0}} \neq \Sigma_{+}^{x_{0}^{\prime}} \forall x_{0}, x_{0}^{\prime} \in M$ with $x_{0} \neq x_{0}^{\prime}$ ), we get that $\mathfrak{B}_{\Sigma}^{-}\left(x_{0}\right)$ and $\mathfrak{B}_{\Sigma}^{-}\left(x_{0}^{\prime}\right)$ must be contained in two different equivalence classes related to the equivalence relation $\sim$. This, in turn, implies that $\left\{\mathfrak{B}_{\Sigma}^{-}\left(x_{0}\right) \mid x_{0} \in M\right\}$ is the exact partition ${ }^{2}$ of $\mathfrak{B}_{\Sigma}^{-}$related to the equivalence relation $\sim$. Therefore, we have $\mathfrak{S}_{\Sigma}=\left\{\mathfrak{B}_{\Sigma}^{-}\left(x_{0}\right) \mid x_{0} \in M\right\}$ and the map $F: x_{0} \mapsto \mathfrak{B}_{\Sigma}^{-}\left(x_{0}\right)$ is a bijection from $M$ to $\mathfrak{S}_{\Sigma}$.

COROLlary 2.12. If the system $\Sigma$ defined by (2.7) is a linear time invariant (LTI) system, i.e., $\dot{x}=f(x, u)=A x+B u$ and $y=h(x, u)=C x+D u$, where $x(t) \in M=\mathbb{R}^{n}, u(t) \in \mathbb{R}^{m}$, and $y(t) \in \mathbb{R}^{p}$ for any $t \in \mathbb{R}$, and $A, B, C, D$ are appropriate dimensional matrixes. Suppose that $(A, C)$ is observable [49], i.e., the $n p \times n$ observability matrix $\left[C^{T},(C A)^{T}, \ldots,\left(C A^{n-1}\right)^{T}\right]^{T}$ is of full column rank $n$. Then there exists a bijective map from $M=\mathbb{R}^{n}$ to $\mathfrak{S}_{\Sigma}$.

Proof. Since $f(x, u)=A x+B u$ is continuous in $u$ and Lipschitz continuous in $x$, this implies that $\Sigma$ is complete. While for the LTI system, that the observability matrix $\left[C^{T},(C A)^{T}, \ldots,\left(C A^{n-1}\right)^{T}\right]^{T}$ has full column rank $n$ implies that the system is forward observable and strongly backward observable. Thus, from Proposition 2.11, there exists a bijective map from $M=\mathbb{R}^{n}$ to $\mathfrak{S}_{\Sigma}$.

2.2.2. Initial conditions for a concrete delay line. To give a further insight into the abstract notion of initial conditions for systems, consider the time delay line model where the output signal is simply a time delayed copy of the input signal. Define input and output signal spaces $\mathcal{U}=\mathcal{Y}=L^{\infty}(\mathbb{R} ; \mathbb{R})$ and $\mathcal{W} \triangleq \mathcal{U} \times \mathcal{Y}$. Then the input-output system of the time $\tau$-delay line model is

$$
\mathfrak{B}_{\tau} \triangleq\left\{(u, y) \in \mathcal{W}_{e} \mid y(t)=u(t-\tau) \forall t \in \mathbb{R}\right\} .
$$

According to (2.1), the set of past trajectories $\mathfrak{B}_{\tau}^{-}$is defined by

$$
\mathfrak{B}_{\tau}^{-}=\left\{\left(u_{-}, y_{-}\right) \in \mathcal{W}_{e}^{-} \mid y_{-}(t)=u_{-}(t-\tau) \forall t \leq 0\right\} .
$$

According to Definition 2.7, the initial state space of $\mathfrak{B}_{\tau}$ is the quotient set $\mathfrak{B}_{\tau}^{-} / \sim$ with the equivalence relation $\sim$ on $\mathfrak{B}_{\tau}^{-}$defined by

$$
w_{-} \sim \tilde{w}_{-} \Leftrightarrow u_{-}(t)=\tilde{u}_{-}(t), \forall t \in[-\tau, 0),
$$

where $w_{-}=\left(u_{-}, y_{-}\right) \in \mathfrak{B}_{\tau}^{-}$and $\tilde{w}_{-}=\left(\tilde{u}_{-}, \tilde{y}_{-}\right) \in \mathfrak{B}_{\tau}^{-}$. And the equivalent class [ $\left.w_{-}\right]$ of any element $w_{-}=\left(u_{-}, y_{-}\right) \in \mathfrak{B}_{\tau}^{-}$is

$$
\left[w_{-}\right]=\left\{\left(\tilde{u}_{-}, \tilde{y}_{-}\right) \in \mathfrak{B}_{\tau}^{-}:\left.\tilde{u}_{-}\right|_{[-\tau, 0)}=\left.u_{-}\right|_{[-\tau, 0)}\right\} .
$$

\footnotetext{
${ }^{2}$ Given any set $X$, let $N$ be a subsets of $X$. Then $N$ is called a partition of $X$ if, and only if, the empty set $\emptyset \notin N$ and $\bigcup_{A \in N}\{A\}=X$, and $A \cap B=\emptyset$ if $A \in N, B \in N$ with $A \neq B$.
} 
The real-valued function $\chi$ on $\mathfrak{B}_{\tau}^{-} / \sim$ is defined by

$$
\left[w_{-}\right] \mapsto \chi\left(\left[w_{-}\right]\right) \triangleq \inf \left\{\left\|\tilde{w}_{-}\right\|: \tilde{w}_{-} \in\left[w_{-}\right]\right\} .
$$

According to (2.5) and (2.4), let $s_{0} \in \mathfrak{B}_{\tau}^{-} / \sim$ be any initial state of $\mathfrak{B}_{\tau}$, and let $u_{+} \in \mathcal{U}_{e}^{+}$denote the future input signal of $\mathfrak{B}_{\tau}$, and let $y_{+} \in \mathcal{Y}_{e}^{+}$denote the future output signal of $\mathfrak{B}_{\tau}$, and then we have

$$
y_{+}(t)=\left(Q_{\tau}^{s_{0}}\left(u_{+}\right)\right)(t) \triangleq \begin{cases}u_{-}(t-\tau) & \text { for } t \in[0, \tau), \\ u_{+}(t-\tau) & \text { for } t \geq \tau,\end{cases}
$$

where $w_{-}=\left(u_{-}, y_{-}\right) \in \mathfrak{B}_{\tau}^{-}$is any element in $s_{0}$.

We know that the time $\tau$-delay line model is an abstract linear system, i.e., a quadruple $(\mathbb{T}, \Phi, \Psi, \mathbb{F})$ defined in Weiss [36, p. 831]. Let the classical state space be $X=L^{\infty}([-\tau, 0) ; \mathbb{R}) ;$ if $x_{t}$ denotes the classical state at time $t \geq 0$, and $u_{+} \in \mathcal{U}_{e}^{+}$ (note that $T_{[0, t)} u_{+} \in \mathcal{U}^{+}$in this example) and $y_{+} \in \mathcal{Y}_{e}^{+}$are the future input signal and output signal, respectively, then

$$
\left(\begin{array}{c}
x_{t} \\
T_{[0, t)} y_{+}
\end{array}\right)=\left(\begin{array}{cc}
\mathbb{T}_{t} & \Phi_{t} \\
\Psi_{t} & \mathbb{F}_{t}
\end{array}\right) \cdot\left(\begin{array}{c}
x(0) \\
T_{[0, t)} u_{+}
\end{array}\right)
$$

Thus, we obtain

$$
y_{+}(t)= \begin{cases}x_{0}(t-\tau) & \text { for } t \in[0, \tau) \\ u_{+}(t-\tau) & \text { for } t \geq \tau\end{cases}
$$

We know by comparing $(2.16)$ and (2.17) that the initial state space $\mathfrak{B}_{\tau}^{-} / \sim$ is actually equivalent to $X=L^{\infty}([-\tau, 0) ; \mathbb{R})$.

2.3. Notion of stability. Given normed signal spaces $\mathcal{U}, \mathcal{Y}$ and $\mathcal{W} \triangleq \mathcal{U} \times \mathcal{Y}$, consider a system $Q$ with initial state space $\mathfrak{S}_{Q}$ at initial time 0 (see Definition 2.7). Suppose that the system $Q$ is well-posed; then we know $Q^{x_{0}}$ is an operator from $\mathcal{U}_{e}^{+}$ to $\mathcal{Y}_{e}^{+}$for any $x_{0} \in \mathfrak{S}_{Q}$. Moreover, if the system $Q$ is causal, so also is $Q^{x_{0}}$. It is easy to see that $\mathfrak{B}_{Q}=\cup_{x_{0} \in \mathfrak{S}_{Q}}\left\{w_{-} \wedge\left(u_{+}, Q^{x_{0}} u_{+}\right) \mid w_{-} \in x_{0}, u_{+} \in \mathcal{U}_{e}^{+}\right\}$. Thus, we can regard the system $Q$ as a family of operators $\left\{Q^{x_{0}}: x_{0} \in \mathfrak{S}_{Q}\right\}$ indexed by initial states.

DEFINITION 2.13. The system $Q$ is said to be input to output stable if and only if it is well-posed and causal, and there exist functions ${ }^{3} \beta \in \mathcal{K} \mathcal{L}$ and $\gamma \in \mathcal{K}_{\infty}$ such that, $\forall x_{0} \in \mathfrak{S}_{Q}, \forall t>0, \forall u_{0+} \in \mathcal{U}^{+}$, we have $\left|\left(Q^{x_{0}} u_{0+}\right)(t)\right| \leq \beta\left(\chi\left(x_{0}\right), t\right)+\gamma\left(\left\|u_{0+}\right\|_{[0, t)}\right)$, where the real-valued functional $\chi(\cdot)$ is defined by $(2.6)$.

The above ISS-like definition represents a generalization of ISS introduced in [27] (see also, e.g., $[28,31,33]$ ) for the system $\dot{x}=f(x, u), y=x$ wherein the term $\beta\left(\chi\left(x_{0}\right), t\right)$ is replaced by $\beta\left(\left\|x_{0}\right\|, t\right)$ in Sontag's definition, and where $x_{0}$ is the initial state $x_{0}=x(0) \in \mathbb{R}^{n}$ rather than the abstract initial condition developed here, which is appropriate for the more general system classes under consideration. More generally, the concept of input-to-output stability (IOS) [33] permits the more general output map $y=h(x)$.

\footnotetext{
${ }^{3}$ A function $\gamma:[0, a) \rightarrow[0, \infty)$ is said to be of class $\mathcal{K}$ if it is continuous and strictly increasing and satisfies $\gamma(0)=0$; moreover, if $a=\infty$ and $\lim _{s \rightarrow \infty} \gamma(s)=\infty$, then it is said to be of class $\mathcal{K}_{\infty}$. A function $\beta:[0, a) \times \mathbb{R}_{+} \rightarrow[0, \infty)$ is said to be of class $\mathcal{K} \mathcal{L}$ if it is such that $\beta(\cdot, t) \in \mathcal{K}$ for each fixed $t \in \mathbb{R}_{+}$, and the function $\beta(s, \cdot)$ is decreasing and $\lim _{t \rightarrow \infty} \beta(s, t)=0$ for each fixed $s \in[0, a)$.
} 


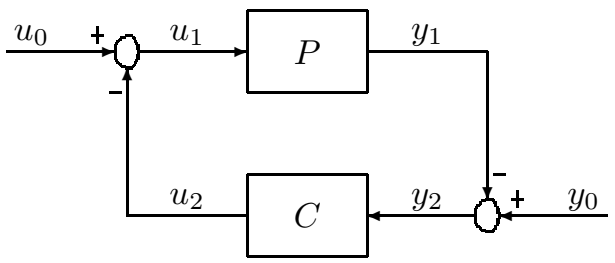

FIG. 1. Closed-loop system $[P, C]$.

2.4. Closed-loop systems. Consider the standard feedback configuration depicted in Figure 1 with the following equations:

$$
[P, C]: \quad w_{i}=\left(u_{i}, y_{i}\right)(i=0,1,2), \quad w_{0}=w_{1}+w_{2},
$$

where $\left(u_{0}, y_{0}\right)$ denote external disturbance, $\left(u_{1}, y_{1}\right)$ are the input-output pairs of the plant $P$ to be controlled, and $\left(u_{2}, y_{2}\right)$ are the output-input pairs of the controller $C$.

Definition 2.14. Given normed signal spaces $\mathcal{U}, \mathcal{Y}, \mathcal{W} \triangleq \mathcal{U} \times \mathcal{Y}$. Let the plant $P$ and the controller $C$ be represented by the sets $\mathfrak{B}_{P}$ and $\mathfrak{B}_{C}$, respectively. ${ }^{4}$ We define the closed-loop system $[P, C]$ by the following set $\mathfrak{B}_{P / / C}$, which is the interconnection of the plant $P$ and controller $C$ shown in Figure 1 that satisfies (2.18),

$$
\mathfrak{B}_{P / / C} \triangleq\left\{\left(w_{0}, w_{1}\right) \in \mathcal{W}_{e} \times \mathcal{W}_{e} \mid w_{1} \in \mathfrak{B}_{P}, w_{2} \triangleq w_{0}-w_{1} \in \mathfrak{B}_{C}\right\} .
$$

In $\mathfrak{B}_{P / / C}$ we view the external input $w_{0}$ as the (closed-loop) input and the internal signal $w_{1}$ as the (closed-loop) output. For the set $\mathfrak{B}_{P / / C}$, we can define the initial state space at initial time 0 of $\mathfrak{B}_{P / / C}$ in terms of Definition 2.7, i.e., let $\mathfrak{B}_{P / / C}^{-} \triangleq R_{-} \mathfrak{B}_{P / / C}$; we similarly define an equivalence relation $\sim$ on $\mathfrak{B}_{P / / C}^{-}$as (2.3), and the set of all equivalence classes $\mathfrak{B}_{P / / C}^{-} / \sim$ is denoted as $\mathfrak{S}_{P / / C}$, which we call initial state space of $\mathfrak{B}_{P / / C}$ at initial time 0 . The size of any initial state in $\mathfrak{S}_{P / / C}$ is similarly defined by (2.6). We next seek to establish the relationship between the initial conditions of the interconnected system, $\mathfrak{S}_{P / / C}$, the plant, $\mathfrak{S}_{P}$, and the controller, $\mathfrak{S}_{C}$.

2.4.1. Initial conditions for the closed-loop and its subsystems. For the classical state space model, it is natural to define the initial state of the closed-loop system by Cartesian product of the initial states of corresponding subsystems. In the following, we will give some answer about the relation between $\mathfrak{S}_{P / / C}$ and $\mathfrak{S}_{P} \times \mathfrak{S}_{C}$. Suppose that the size of any $x_{0}=\left(x_{10}, x_{20}\right) \in \mathfrak{S}_{P} \times \mathfrak{S}_{C}$ is defined in the usual way, e.g., for an appropriate $q \in[1, \infty]$,

$$
\chi\left(x_{0}\right) \triangleq\left(\chi\left(x_{10}\right)^{q}+\chi\left(x_{20}\right)^{q}\right)^{\frac{1}{q}}=\inf \left\{\left\|\left(w_{1-}, w_{2-}\right)\right\| \mid w_{1-} \in x_{10}, w_{2-} \in x_{20}\right\}
$$

Note that for any $s_{0} \in \mathfrak{S}_{P / / C}$ and $w_{0+} \in \mathcal{W}_{e}^{+}$, we have defined a set $\Pi_{P / / C}^{s_{0}}\left(w_{0+}\right)$ according to (2.5) and (2.4) (let $\mathfrak{B}_{Q}=\mathfrak{B}_{P / / C}$ and $\Pi_{P / / C}^{s_{0}}\left(w_{0+}\right)=Q^{s_{0}}\left(w_{0+}\right)$ ), i.e.,

$$
\Pi_{P / / C}^{s_{0}}\left(w_{0+}\right) \triangleq\left\{w_{1+} \in \mathcal{W}_{e}^{+} \mid \begin{array}{c}
\left(w_{0-}, w_{1-}\right) \wedge\left(w_{0+}, w_{1+}\right) \in \mathfrak{B}_{P / / C} \\
\forall\left(w_{0-}, w_{1-}\right) \in s_{0}
\end{array}\right\} .
$$

To understand the relation between $\mathfrak{S}_{P / / C}$ and $\mathfrak{S}_{P} \times \mathfrak{S}_{C}$, we need to define another

\footnotetext{
${ }^{4}$ Note that when considering the controller $C$, we need interchange the role of $\mathcal{U}_{e}$ and $\mathcal{Y}_{e}$ and think of $y_{2} \in \mathcal{Y}_{e}$ as the input and $u_{2} \in \mathcal{U}_{e}$ as the output.
}

Copyright $@$ by SIAM. Unauthorized reproduction of this article is prohibited. 
set which is related to the product state $\mathfrak{S}_{P} \times \mathfrak{S}_{C}$, denoted by $\overline{\Pi_{P / / C}^{x_{0}}}\left(w_{0+}\right)$, for any $x_{0}=\left(x_{10}, x_{20}\right) \in \mathfrak{S}_{P} \times \mathfrak{S}_{C}$ and any $w_{0+} \in \mathcal{W}_{e}^{+}$, as follows: ${ }^{5}$

$$
\overline{\Pi_{P / / C}^{x_{0}}}\left(w_{0+}\right) \triangleq\left\{\begin{array}{c|c}
w_{1+} \in \mathcal{W}_{e}^{+} & \begin{array}{c}
\left(w_{0-}, w_{1-}\right) \wedge\left(w_{0+}, w_{1+}\right) \in \mathfrak{B}_{P / / C} \\
\forall\left(w_{1-}, w_{0-}-w_{1-}\right) \in x_{0}
\end{array}
\end{array}\right\} .
$$

THEOREM 2.15. There exists a surjective and bounded ${ }^{6}$ map $\pi: \mathfrak{S}_{P} \times \mathfrak{S}_{C} \rightarrow$ $\mathfrak{S}_{P / / C}$ such that $\overline{\Pi_{P / / C}^{x_{0}}}\left(w_{0+}\right)=\Pi_{P / / C}^{\pi\left(x_{0}\right)}\left(w_{0+}\right) \forall x_{0} \in \mathfrak{S}_{P} \times \mathfrak{S}_{C}$ and $\forall w_{0+} \in \mathcal{W}_{e}^{+}$. If we define an equivalence relation $\stackrel{\pi}{\sim}$ on $\mathfrak{S}_{P} \times \mathfrak{S}_{C}$ by $x_{0} \stackrel{\pi}{\sim} y_{0} \Leftrightarrow \pi\left(x_{0}\right)=\pi\left(y_{0}\right)$, and the equivalence class $\left[x_{0}\right] \triangleq\left\{y_{0} \in \mathfrak{S}_{P} \times \mathfrak{S}_{C} \mid y_{0} \stackrel{\pi}{\sim} x_{0}\right\}$, and the size $\chi\left(\left[x_{0}\right]\right) \triangleq$ $\inf \left\{\chi\left(y_{0}\right) \mid y_{0} \in\left[x_{0}\right]\right\}$, and another map $\bar{\pi}$ induced by $\pi$ as

$$
\bar{\pi}:\left(\mathfrak{S}_{P} \times \mathfrak{S}_{C}\right) / \underset{\sim}{\sim} \rightarrow \mathfrak{S}_{P / / C}, \quad \bar{\pi}\left(\left[x_{0}\right]\right)=\pi\left(x_{0}\right),
$$

then $\bar{\pi}$ is a bijective and bounded map, and the inverse $\bar{\pi}^{-1}$ is also bounded.

Proof. For any $x_{0}=\left(x_{10}, x_{20}\right) \in \mathfrak{S}_{P} \times \mathfrak{S}_{C}$, choose any $w_{1-} \in x_{10}, w_{2-} \in x_{20}$ and define $w_{0-} \triangleq w_{1-}+w_{2-}$. From Definitions 2.7 and 2.14 , we get $s_{0} \triangleq\left[\left(w_{0-}, w_{1-}\right)\right] \in$ $\mathfrak{S}_{P / / C}$. Next, we show that $s_{0}$ is independent of the choice of $w_{1-} \in x_{10}, w_{2-} \in x_{20}$.

Choose any other $w_{1-}^{\prime} \in x_{10}, w_{2-}^{\prime} \in x_{20}$ and define $w_{0-}^{\prime}=w_{1_{-}}^{\prime}+w_{2-}^{\prime}$; thus, we have $s_{0}^{\prime} \triangleq\left[\left(w_{0-}^{\prime}, w_{1-}^{\prime}\right)\right] \in \mathfrak{S}_{P / / C}$. We need to show $s_{0}^{\prime}=s_{0}$. According to (2.3) and the definition of the equivalence class (see section 2.2), this is equivalent to saying $\Pi_{P / / C}^{\left(w_{0-}, w_{1-}\right)}\left(w_{0+}\right)=\Pi_{P / / C}^{\left(w_{0-}^{\prime}, w_{1-}^{\prime}\right)}\left(w_{0+}\right)$ for any $w_{0+} \in \mathcal{W}_{e}^{+}$. In order to prove these equalities, by symmetry, we only need to show $\Pi_{P / / C}^{\left(w_{0-}, w_{1-}\right)}\left(w_{0+}\right) \subseteq$ $\Pi_{P / / C}^{\left(w_{0-}^{\prime}, w_{1-}^{\prime}\right)}\left(w_{0+}\right) \forall w_{0+} \in \mathcal{W}_{e}^{+}$. To this end, for any $w_{1+} \in \Pi_{P / / C}^{\left(w_{0-}, w_{1-}\right)}\left(w_{0+}\right)$, we define $w_{2+}=w_{0+}-w_{1+}$, and thus from Definition 2.14 we have $w_{1-} \wedge w_{1+} \in \mathfrak{B}_{P}$ and $w_{2-} \wedge w_{2+} \in \mathfrak{B}_{C}$. Since both $w_{1-}$ and $w_{1-}^{\prime}$ belong to $x_{10}$, we have from the definition of initial conditions for $P$ that $P^{w_{1-}}\left(u_{1+}\right)=P^{w_{1-}^{\prime}}\left(u_{1+}\right) \forall u_{1+} \in \mathcal{U}_{e}^{+}$. This implies that $w_{1-}^{\prime} \wedge w_{1+} \in \mathfrak{B}_{P}$. By a similar argument, we also have $w_{2-}^{\prime} \wedge w_{2+} \in \mathfrak{B}_{C}$. Thus, from Definition 2.14, we obtain $\left(w_{0-}^{\prime} \wedge w_{0+}, w_{1-}^{\prime} \wedge w_{1+}\right) \in \mathfrak{B}_{P / / C}$. This implies that $w_{1+} \in \Pi_{P / / C}^{\left(w_{0-}^{\prime}, w_{1-}^{\prime}\right)}\left(w_{0+}\right)$ and thus $\Pi_{P / / C}^{\left(w_{0-}, w_{1-}\right)}\left(w_{0+}\right) \subseteq \Pi_{P / / C}^{\left(w_{0-}^{\prime}, w_{1-}^{\prime}\right)}\left(w_{0+}\right)$. Therefore, $s_{0}$ is only related to $x_{10}$ and $x_{20}$. We also have $\overline{\Pi_{P / / C}^{x_{0}}}\left(w_{0+}\right)=\Pi_{P / / C}^{s_{0}}\left(w_{0+}\right)$ for any $w_{0+} \in \mathcal{W}_{e}^{+}$.

A natural map $\pi: \mathfrak{S}_{P} \times \mathfrak{S}_{C} \rightarrow \mathfrak{S}_{P / / C}$ can be defined by $x_{0} \mapsto s_{0}$. From (2.6) and $s_{0}=\left[\left(w_{0-}, w_{1-}\right)\right]$, we have $\chi\left(\pi\left(x_{0}\right)\right)=\chi\left(s_{0}\right) \leq\left\|\left(w_{0-}, w_{1-}\right)\right\|=\|\left(w_{1-}+\right.$ $\left.w_{2-}, w_{1-}\right) \| \leq\left(\left\|w_{1-}+w_{2-}\right\|^{q}+\left\|w_{1-}\right\|^{q}\right)^{1 / q} \leq\left(2^{q}+1\right)^{1 / q}\left(\left\|w_{1-}\right\|^{q}+\left\|w_{2-}\right\|^{q}\right)^{1 / q}$ for any $q \geq 1$. Since $w_{1-}$ and $w_{2-}$ are arbitrarily chosen from $x_{10}$ and $x_{20}$, respectively, we have $\chi\left(\pi\left(x_{0}\right)\right) \leq\left(2^{q}+1\right)^{1 / q} \cdot \chi\left(x_{0}\right)$. This implies that the map $\pi$ is bounded. Next, we show that $\pi$ is also a surjective map. To this end, for any $s_{0}^{\prime \prime} \in \mathfrak{S}_{P / / C}$, choose any $\left(w_{0-}^{\prime \prime}, w_{1-}^{\prime \prime}\right) \in s_{0}^{\prime \prime}$ and define $w_{2-}^{\prime \prime} \triangleq w_{0-}^{\prime \prime}-w_{1-}^{\prime \prime}$; thus, from (2.19) and (2.1), we have $w_{1-}^{\prime \prime} \in \mathfrak{B}_{P}^{-}$and $w_{2-}^{\prime \prime} \in \mathfrak{B}_{C}^{-}$. Define $x_{10}^{\prime \prime} \triangleq\left[w_{1-}^{\prime \prime}\right], x_{20}^{\prime \prime} \triangleq\left[w_{2-}^{\prime \prime}\right]$ and $x_{0}^{\prime \prime} \triangleq\left(x_{10}^{\prime \prime}, x_{20}^{\prime \prime}\right)$, and we have $x_{0}^{\prime \prime} \in \mathfrak{S}_{P} \times \mathfrak{S}_{C}$ and $\pi\left(x_{0}^{\prime \prime}\right)=s_{0}^{\prime \prime}$. This implies that the map $\pi$ is surjective.

\footnotetext{
${ }^{5}$ Note that if $[P, C]$ is well-posed (see section 2.4 .3 below), then $\Pi_{P / / C}^{s_{0}}$ in $(2.21)$ (resp., $\overline{\Pi_{P / / C}^{x_{0}}}$ in $(2.22)$ ) actually defines an operator from $\mathcal{W}_{e}^{+}$to $\mathcal{W}_{e}^{+}$for any initial state $s_{0} \in \mathfrak{S}_{P / / C}$ (resp., $\left.x_{0} \in \mathfrak{S}_{P} \times \mathfrak{S}_{C}\right)$. Moreover, we have a natural surjective map $\pi: \mathfrak{S}_{P} \times \mathfrak{S}_{C} \rightarrow \mathfrak{S}_{P / / C}$ defined in Theorem 2.15 such that $\Pi_{P / / C}^{\pi\left(x_{0}\right)}=\overline{\Pi_{P / / C}^{x_{0}}}$ for any $x_{0} \in \mathfrak{S}_{P} \times \mathfrak{S}_{C}$.

${ }^{6}$ Here, bounded means that there exists a positive number $r \geq 0$ such that $\chi\left(\pi\left(x_{0}\right)\right) \leq r \cdot \chi\left(x_{0}\right)$ for any $x_{0} \in \mathfrak{S}_{P} \times \mathfrak{S}_{C}$ with function $\chi$ defined by (2.6).
}

Copyright $@$ by SIAM. Unauthorized reproduction of this article is prohibited. 
Define a map $\bar{\pi}$ by $(2.23)$, and it is easy to see that $\bar{\pi}$ is bijective. It follows from $\chi\left(\bar{\pi}\left(\left[x_{0}\right]\right)\right)=\chi\left(\pi\left(x_{0}\right)\right) \leq\left(2^{q}+1\right)^{1 / q} \cdot \chi\left(x_{0}\right)$ for any $q \geq 1$ that the map $\bar{\pi}$ is bounded.

Finally, we show that the inverse map $\bar{\pi}^{-1}: \mathfrak{S}_{P / / C} \rightarrow\left(\mathfrak{S}_{P} \times \mathfrak{S}_{C}\right) /_{\sim}$ is also bounded. To this end, for any $s_{0}^{\prime \prime} \in \mathfrak{S}_{P / / C}$, from the proof of map $\pi$ being surjective, we have $\bar{\pi}^{-1}\left(s_{0}^{\prime \prime}\right)=\left[x_{0}^{\prime \prime}\right]$. Thus, by applying $(2.20)$, we get $\chi\left(\bar{\pi}^{-1}\left(s_{0}^{\prime \prime}\right)\right)=\chi\left(\left[x_{0}^{\prime \prime}\right]\right) \leq$ $\chi\left(x_{0}^{\prime \prime}\right) \leq\left(\left\|w_{1-}^{\prime \prime}\right\|^{q}+\left\|w_{2-}^{\prime \prime}\right\|^{q}\right)^{1 / q}=\left(\left\|w_{1-}^{\prime \prime}\right\|^{q}+\left\|w_{0-}^{\prime \prime}-w_{1-}^{\prime \prime}\right\|^{q}\right)^{1 / q} \leq\left(2^{q}+1\right)^{1 / q} \cdot\left(\left\|w_{0-}^{\prime \prime}\right\|^{q}+\right.$ $\left.\left\|w_{1-}^{\prime \prime}\right\|^{q}\right)^{1 / q}$. Since $\left(w_{0-}^{\prime \prime}, w_{1-}^{\prime \prime}\right)$ is arbitrarily chosen from $s_{0}^{\prime \prime}$, we have $\chi\left(\bar{\pi}^{-1}\left(s_{0}^{\prime \prime}\right)\right) \leq$ $\left(2^{q}+1\right)^{1 / q} \cdot \chi\left(s_{0}^{\prime \prime}\right)$. This implies that the inverse map $\bar{\pi}^{-1}$ is also bounded.

2.4.2. State space initial conditions for closed-loop systems. Consider the closed-loop system shown in Figure 1. The forward and feedback loop represent the plant $P$ and controller $C$, respectively. Both $P$ and $C$ with classical initial state spaces $x_{p} \in X_{p}=\mathbb{R}^{n_{p}}$ and $x_{c} \in X_{c}=\mathbb{R}^{n_{c}}$, respectively, are defined like (2.7), i.e., $\dot{x}_{p}=f_{p}\left(x_{p}, u_{1}\right), y_{1}=h_{p}\left(x_{p}, u_{1}\right)$ and $\dot{x}_{c}=f_{c}\left(x_{c}, y_{2}\right), u_{2}=h_{c}\left(x_{c}, y_{2}\right)$. Figure 1 represents the following closed-loop equations:

$$
\begin{aligned}
& \dot{x}_{p}=f_{p}\left(x_{p}, u_{1}\right), \quad \dot{x}_{c}=f_{c}\left(x_{c}, y_{0}-y_{1}\right), \\
& u_{1}=u_{0}-h_{c}\left(x_{c}, y_{0}-y_{1}\right), \quad y_{1}=h_{p}\left(x_{p}, u_{1}\right),
\end{aligned}
$$

with product state space $X_{p} \times X_{c}$ and with $\left(u_{0}, y_{0}\right)$ as inputs and $\left(u_{1}, y_{1}\right)$ as outputs.

With the concepts of complete, forward observable, and strongly backward observable defined by Definitions 2.9 and 2.10 , we have the following.

TheOrem 2.16. Suppose that $P, C$, and the closed-loop (2.24) are complete. If both $P$ and $C$ are forward observable (resp., strongly backward observable), then the closed-loop (2.24) is forward observable (resp., strongly backward observable).

Proof. We establish forward observability of the closed-loop (2.24) by contradiction. It is thus assumed that there exist $\left(x_{p 0}, x_{c 0}\right) \in X_{p} \times X_{c},\left(x_{p 0}^{\prime}, x_{c 0}^{\prime}\right) \in X_{p} \times X_{c}$ with $\left(x_{p 0}, x_{c 0}\right) \neq\left(x_{p 0}^{\prime}, x_{c 0}^{\prime}\right)$ such that

$$
\left.\left(u_{1}, y_{1}\right)\right|_{t \geq 0}=\left.\left.\left(u_{1}^{\prime}, y_{1}^{\prime}\right)\right|_{t \geq 0} \quad \forall\left(u_{0}, y_{0}\right)\right|_{t \geq 0}=\left.\left(u_{0}^{\prime}, y_{0}^{\prime}\right)\right|_{t \geq 0} .
$$

This implies that

$$
\left.\left.\left(y_{1}, u_{2}\right)\right|_{t \geq 0} \triangleq\left(h_{p}\left(x_{p}, u_{1}\right), h_{c}\left(x_{c}, y_{2}\right)\right)\right|_{t \geq 0}=\left.\left.\left(h_{p}\left(x_{p}^{\prime}, u_{1}^{\prime}\right), h_{c}\left(x_{c}^{\prime}, y_{2}^{\prime}\right)\right)\right|_{t \geq 0} \triangleq\left(y_{1}^{\prime}, u_{2}^{\prime}\right)\right|_{t \geq 0}
$$

for any $\left.\left(u_{1}, y_{2}\right)\right|_{t \geq 0}=\left.\left(u_{1}^{\prime}, y_{2}^{\prime}\right)\right|_{t \geq 0}$ which satisfy

$$
\begin{array}{lll}
\dot{x}_{p}=f_{p}\left(x_{p}, u_{1}\right), & \dot{x}_{c}=f_{c}\left(x_{c}, y_{2}\right), & \left(x_{p}(0), x_{c}(0)\right)=\left(x_{p 0}, x_{c 0}\right) ; \\
\dot{x}_{p}^{\prime}=f_{p}\left(x_{p}^{\prime}, u_{1}^{\prime}\right), & \dot{x}_{c}^{\prime}=f_{c}\left(x_{c}^{\prime}, y_{2}^{\prime}\right), & \left(x_{p}^{\prime}(0), x_{c}^{\prime}(0)\right)=\left(x_{p 0}^{\prime}, x_{c 0}^{\prime}\right) .
\end{array}
$$

To this end, let $u_{0}=u_{1}+u_{2}, u_{0}^{\prime}=u_{1}^{\prime}+u_{2}^{\prime}, y_{0}=y_{1}+y_{2}$, and $y_{0}^{\prime}=y_{1}^{\prime}+y_{2}^{\prime}$. It follows from the completeness of $P$ that $u_{0}$ (resp., $u_{0}^{\prime}$ ) is uniquely determined by $u_{1}$ and $x_{p 0}$ (resp., $u_{1}^{\prime}$ and $x_{p 0}^{\prime}$ ). Similarly, $y_{0}$ (resp., $y_{0}^{\prime}$ ) is uniquely determined by $y_{2}$ and $x_{c 0}$ (resp., $y_{2}^{\prime}$ and $x_{c 0}^{\prime}$ ) by using the completeness of $C$. Since the closed-loop (2.24) is also complete, we know that for $\left(u_{0}^{\prime \prime}, y_{0}^{\prime \prime}\right)=\left(u_{0}, y_{0}\right)$ and $\left(x_{p}^{\prime \prime}(0), x_{c}^{\prime \prime}(0)\right)=\left(x_{p 0}^{\prime}, x_{c 0}^{\prime}\right)$ there exist unique $x_{p}^{\prime \prime}, x_{c}^{\prime \prime}, u_{1}^{\prime \prime}, y_{1}^{\prime \prime}, u_{2}^{\prime \prime}, y_{2}^{\prime \prime}$ satisfying

$$
\begin{array}{rll}
\dot{x}_{p}^{\prime \prime}=f_{p}\left(x_{p}^{\prime \prime}, u_{1}^{\prime \prime}\right), & y_{1}^{\prime \prime}=h_{p}\left(x_{p}^{\prime \prime}, u_{1}^{\prime \prime}\right), & u_{0}^{\prime \prime}=u_{1}^{\prime \prime}+u_{2}^{\prime \prime} ; \\
\dot{x}_{c}^{\prime \prime}=f_{c}\left(x_{c}^{\prime \prime}, y_{2}^{\prime \prime}\right), & u_{2}^{\prime \prime}=h_{c}\left(x_{c}^{\prime \prime}, y_{2}^{\prime \prime}\right), & y_{0}^{\prime \prime}=y_{1}^{\prime \prime}+y_{2}^{\prime \prime} .
\end{array}
$$

From (2.25), we must have $\left.\left(u_{i}^{\prime \prime}, y_{i}^{\prime \prime}\right)\right|_{t \geq 0}=\left.\left(u_{i}, y_{i}\right)\right|_{t \geq 0}$ for $i=0,1,2$, and thus $\left(u_{1}^{\prime \prime}\right.$, $\left.y_{2}^{\prime \prime}\right)\left.\right|_{t \geq 0}=\left.\left(u_{1}, y_{2}\right)\right|_{t \geq 0}=\left.\left(u_{1}^{\prime}, y_{2}^{\prime}\right)\right|_{t \geq 0}$. Since $\left(u_{0}^{\prime}, y_{0}^{\prime}\right)$ are uniquely determined by 
$\left(u_{1}^{\prime}, y_{2}^{\prime}\right)$ and $\left(x_{p 0}^{\prime}, x_{c 0}^{\prime}\right)$ (see above), we have $\left.\left(u_{0}^{\prime \prime}, y_{0}^{\prime \prime}\right)\right|_{t \geq 0}=\left.\left(u_{0}^{\prime}, y_{0}^{\prime}\right)\right|_{t \geq 0}$, and thus $\left.\left(u_{i}^{\prime \prime}, y_{i}^{\prime \prime}\right)\right|_{t \geq 0}=\left.\left(u_{i}^{\prime}, y_{i}^{\prime}\right)\right|_{t \geq 0}$ for $i=0,1,2$. This in turn implies that $\left.\left(u_{i}^{\prime}, y_{i}^{\prime}\right)\right|_{t \geq 0}=$ $\left.\left(u_{i}^{\prime \prime}, y_{i}^{\prime \prime}\right)\right|_{t \geq 0}=\left.\left(u_{i}, y_{i}\right)\right|_{t \geq 0}$ for $i=0,1,2$, and the required result $\left.\left(y_{1}, u_{2}\right)\right|_{t \geq 0}=$ $\left.\left(y_{1}^{\prime}, u_{2}^{\prime}\right)\right|_{t \geq 0}$ follows.

Since $\left.\left(u_{1}, y_{2}\right)\right|_{t \geq 0}=\left.\left(u_{1}^{\prime}, y_{2}^{\prime}\right)\right|_{t \geq 0}$ in (2.26) can thus be taken as any element by choosing $u_{0}=u_{1}+h_{c}\left(x_{c}, y_{2}\right)$ and $y_{0}=y_{2}+h_{p}\left(x_{p}, u_{1}\right)$ with $\dot{x}_{p}=f_{p}\left(x_{p}, u_{1}\right)$, $\dot{x}_{c}=f_{c}\left(x_{c}, y_{2}\right)$, and $\left(x_{p}(0), x_{x}(0)\right)=\left(x_{p 0}, x_{c 0}\right)$, we obtain that for the above given $\left(x_{p 0}, x_{c 0}\right) \neq\left(x_{p 0}^{\prime}, x_{c 0}^{\prime}\right)$ we have $\left.\left(y_{1}, u_{2}\right)\right|_{t \geq 0}=\left.\left(y_{1}^{\prime}, u_{2}^{\prime}\right)\right|_{t \geq 0}$ for any $\left.\left(u_{1}, y_{2}\right)\right|_{t \geq 0}=$ $\left.\left(u_{1}^{\prime}, y_{2}^{\prime}\right)\right|_{t \geq 0}$. This contradicts forward observability of $P$ and $C$. Thus, the closedloop (2.24) is forward observable.

We also show strongly backward observability of the closed-loop (2.24) by contradiction. Assume, therefore, that there exist $\left(x_{p 0}, x_{c 0}\right) \in X_{p} \times X_{c},\left(x_{p 0}^{\prime}, x_{c 0}^{\prime}\right) \in X_{p} \times X_{c}$ with $\left(x_{p 0}, x_{c 0}\right) \neq\left(x_{p 0}^{\prime}, x_{c 0}^{\prime}\right)$ and $\left.\left(u_{0}, y_{0}\right)\right|_{t \leq 0}=\left.\left(u_{0}^{\prime}, y_{0}^{\prime}\right)\right|_{t \leq 0}$ such that $\left.\left(u_{1}, y_{1}\right)\right|_{t \leq 0}=$ $\left.\left(u_{1}^{\prime}, y_{1}^{\prime}\right)\right|_{t \leq 0}$, and thus $\left.\left(u_{2}, y_{2}\right)\right|_{t \leq 0}=\left.\left(u_{2}^{\prime}, y_{2}^{\prime}\right)\right|_{t \leq 0}$. This implies that there exist $\left.u_{1}\right|_{t \leq 0}=$ $\left.u_{1}^{\prime}\right|_{t \leq 0}$ and $\left.y_{2}\right|_{t \leq 0}=\left.y_{2}^{\prime}\right|_{t \leq 0}$ such that $\left.y_{1}\right|_{t \leq 0}=\left.y_{1}^{\prime}\right|_{t \leq 0}$ and $\left.u_{2}\right|_{t \leq 0}=\left.u_{2}^{\prime}\right|_{t \leq 0}$. This is a contradiction to the fact that both $P$ and $C$ are strongly backward observable. Thus, the closed-loop (2.24) is strongly backward observable.

Consider the set $\mathfrak{B}_{P / / C}$ which consists of all input-output pairs $\left(\left(u_{0}, y_{0}\right),\left(u_{1}, y_{1}\right)\right)$ satisfying (2.24). Using the same procedure as in section 2.2, the initial state space $\mathfrak{S}_{P / / C}$ (see Definition 2.7) for the set $\mathfrak{B}_{P / / C}$ at initial time 0 can be defined.

Theorem 2.17. Suppose that $P, C$, and the closed-loop (2.24) are complete. If both $P$ and $C$ are forward observable and strongly backward observable, then there exists a bijective map from $X_{p} \times X_{c}$ to $\mathfrak{S}_{P / / C}$.

Proof. This follows directly from Theorem 2.16 and Proposition 2.11.

2.4.3. Well-posedness and stability of closed-loop systems. Since the closed-loop system $[P, C]$ represented by $\mathfrak{B}_{P / / C}$ is a system in terms of Definition 2.1, we can similarly define the existence, uniqueness, and well-posedness of $\mathfrak{B}_{P / / C}$ by Definition 2.4, wherein $w_{0} \in \mathcal{W}_{e}$ is the input and $w_{1} \in \mathcal{W}_{e}$ is the output. That is, the closed-loop system $[P, C]$ has the existence property if for any $s_{0} \in \mathfrak{S}_{P / / C}$ and any $w_{0+} \in \mathcal{W}_{e}^{+}$, there exists a $w_{1+} \in \mathcal{W}_{e}^{+}$such that $w_{1+} \in \Pi_{P / / C}^{s_{0}}\left(w_{0+}\right)$ with $\Pi_{P / / C}^{s_{0}}\left(w_{0+}\right)$ defined by (2.21), it has the uniqueness property if $\forall s_{0} \in \mathfrak{S}_{P / / C}$ and all $w_{0+} \in \mathcal{W}_{e}^{+}$, $w_{1+}, \tilde{w}_{1+} \in \Pi_{P / / C}^{s_{0}}\left(w_{0+}\right) \Rightarrow w_{1+}=\tilde{w}_{1+}$, and it is well-posed if it has both the existence and uniqueness properties. Note that by Theorem 2.15, it also follows that $s_{0} \in \mathfrak{S}_{P / / C}$ and $\Pi_{P / / C}^{s_{0}}$ can be replaced throughout in the above by $s_{0} \in \mathfrak{S}_{P} \times \mathfrak{S}_{C}$ and $\overline{\Pi_{P / / C}^{s_{0}}}$, respectively.

Following directly from Definition 2.13, we define input-to-output stability for the closed-loop system $[P, C]$.

Definition 2.18. The closed-loop system $[P, C]$ with initial state space $\mathfrak{S}_{P / / C}$ is said to be input to output stable if and only if it is well-posed and causal, and there exist functions $\beta \in \mathcal{K} \mathcal{L}$ and $\gamma \in \mathcal{K}_{\infty}$ such that $\forall s_{0} \in \mathfrak{S}_{P / / C}, \forall t>0, \forall w_{0+} \in \mathcal{W}^{+}$,

$$
\left|\left(\Pi_{P / / C}^{s_{0}} w_{0+}\right)(t)\right| \leq \beta\left(\chi\left(s_{0}\right), t\right)+\gamma\left(\left\|w_{0+}\right\|_{[0, t)}\right) .
$$

Note that two well-posed open subsystems (plant and controller) does not necessarily result in a well-posed closed-loop system and that the causality of a closed-loop system doesn't follow from the causality of open-loop subsystems (plant and controller) [37, section 4.3.2]. The following theorem gives an alternative characterization of the property of input to output stable for a closed-loop system. 
TheOREm 2.19. Suppose that the closed-loop system $[P, C]$ is well-posed and causal. The following four statements are equivalent:

I. The closed-loop system $[P, C]$ is input to output stable.

II. There exist $\beta_{1} \in \mathcal{K} \mathcal{L}$ and $\gamma_{1} \in \mathcal{K}_{\infty}$ such that $\forall s_{0} \in \mathfrak{S}_{P / / C}, \forall t>0, \forall w_{0+} \in$ $\mathcal{W}^{+}$

$$
\left|\left(\Pi_{P / / C}^{s_{0}} w_{0+}\right)(t)\right| \leq \beta_{1}\left(\chi\left(s_{0}\right), t\right)+\gamma_{1}\left(\left\|w_{0+}\right\|_{[0, t)}\right) .
$$

III. There exist $\beta_{2} \in \mathcal{K} \mathcal{L}, \gamma_{2} \in \mathcal{K}_{\infty}$ such that $\forall x_{0} \in \mathfrak{S}_{P} \times \mathfrak{S}_{C}, \forall t>0, \forall w_{0+} \in$ $\mathcal{W}^{+}$

$$
\left|\left(\overline{\Pi_{P / / C}^{x_{0}}} w_{0+}\right)(t)\right| \leq \beta_{2}\left(\chi\left(x_{0}\right), t\right)+\gamma_{2}\left(\left\|w_{0+}\right\|_{[0, t)}\right) .
$$

IV. There exist $\beta_{3} \in \mathcal{K} \mathcal{L}$ and $\gamma_{3} \in \mathcal{K}_{\infty}$ such that $\forall x_{0}=\left(x_{10}, x_{20}\right) \in \mathfrak{S}_{P} \times$ $\mathfrak{S}_{C}, \forall t>0, \forall w_{0+} \in \mathcal{W}^{+}, \forall w_{1-} \in x_{10}, \forall w_{2-} \in x_{20}$,

$$
\left|\left(\overline{\Pi_{P / / C}^{x_{0}}} w_{0+}\right)(t)\right| \leq \beta_{3}\left(\left\|\left(w_{1-}, w_{2-}\right)\right\|, t\right)+\gamma_{3}\left(\left\|w_{0+}\right\|_{[0, t)}\right) .
$$

Moreover, we have $\gamma_{1}=\gamma_{2}=\gamma_{3}$ and $\beta_{2}=\beta_{3}$.

Proof. I $\Leftrightarrow$ II: This follows from Definition 2.18.

II $\Rightarrow$ III: Suppose that (2.27) holds with given functions $\beta_{1} \in \mathcal{K} \mathcal{L}, \gamma_{1} \in \mathcal{K}_{\infty}$. For any $x_{0} \in \mathfrak{S}_{P} \times \mathfrak{S}_{C}$, by Theorem 2.15 , we have $\pi\left(x_{0}\right) \in \mathfrak{S}_{P / / C}$ and $\overline{\Pi_{P / / C}^{x_{0}}}=\Pi_{P / / C}^{\pi\left(x_{0}\right)}$, and $\chi\left(\pi\left(x_{0}\right)\right) \leq\|\pi\| \cdot \chi\left(x_{0}\right)$. (Note that $\pi$ is a bounded map.) Define a function $\beta_{2} \in \mathcal{K} \mathcal{L}$ by $\beta_{2}(r, t) \triangleq \beta_{1}(\|\pi\| r, t) \forall r \geq 0, t \geq 0$. We have that (2.28) holds with $\gamma_{2}=\gamma_{1}$.

III $\Rightarrow$ II: Suppose that (2.28) holds with given functions $\beta_{2} \in \mathcal{K} \mathcal{L}$ and $\gamma_{2} \in$ $\mathcal{K}_{\infty}$. For any $s_{0} \in \mathfrak{S}_{P / / C}$, by Theorem 2.15 , we have $\bar{\pi}^{-1}\left(s_{0}\right) \in\left(\mathfrak{S}_{P} \times \mathfrak{S}_{C}\right) / \pi$ and $\chi\left(\bar{\pi}^{-1}\left(s_{0}\right)\right) \leq\left\|\bar{\pi}^{-1}\right\| \chi\left(s_{0}\right)$. (Note that $\bar{\pi}^{-1}$ is a bounded bijective map.) For any $\tilde{\varepsilon}>0$, there exists an $x_{0} \in \mathfrak{S}_{P} \times \mathfrak{S}_{C}$ such that $x_{0} \in \bar{\pi}^{-1}\left(s_{0}\right)$ and $\chi\left(x_{0}\right) \leq \chi\left(\bar{\pi}^{-1}\left(s_{0}\right)\right)+\varepsilon$.

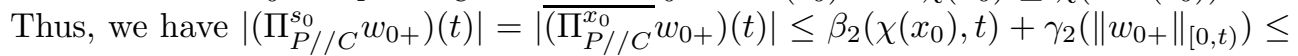
$\beta_{2}\left(\left\|\bar{\pi}^{-1}\right\| \cdot \chi\left(s_{0}\right)+\varepsilon, t\right)+\gamma_{2}\left(\left\|w_{0+}\right\|_{[0, t)}\right)$ for any $t>0$ and any $w_{0+} \in \mathcal{W}^{+}$. Since $\varepsilon$ is an arbitrarily chosen positive number, we have that (2.27) holds with $\gamma_{1}=\gamma_{2}$ and $\beta_{1}(r, t)=\beta_{2}\left(\left\|\bar{\pi}^{-1}\right\| \cdot r, t\right) \forall r \geq 0$ and $t \geq 0$.

III $\Rightarrow$ IV: Suppose that (2.28) holds with given functions $\beta_{2} \in \mathcal{K} \mathcal{L}$ and $\gamma_{2} \in \mathcal{K}_{\infty}$. From $(2.20)$, we know that $\chi\left(x_{0}\right) \leq\left\|\left(w_{1-}, w_{2-}\right)\right\|$ for any $w_{1-} \in x_{10}$ and any $w_{2-} \in$ $x_{20}$. Thus, we have that (2.29) holds with $\beta_{3}=\beta_{2}$ and $\gamma_{3}=\gamma_{2}$.

IV $\Rightarrow$ III: Suppose that (2.29) holds with given functions $\beta_{3} \in \mathcal{K} \mathcal{L}$ and $\gamma_{3} \in \mathcal{K}_{\infty}$. For any $x_{0}=\left(x_{10}, x_{20}\right) \in \mathfrak{S}_{P} \times \mathfrak{S}_{C}$, for any $\varepsilon>0$, from (2.20) we know that there exist $w_{1-} \in x_{10}$ and $w_{2-} \in x_{20}$ such that $\left\|\left(w_{1-}, w_{2-}\right)\right\| \leq \chi\left(x_{0}\right)+\varepsilon$. Thus, we have $\left|\left(\overline{\Pi_{P / / C}^{x_{0}}} w_{0+}\right)(t)\right| \leq \beta_{3}\left(\left\|\left(w_{1-}, w_{2-}\right)\right\|, t\right)+\gamma_{3}\left(\left\|w_{0+}\right\|_{[0, t)}\right) \leq \beta_{3}\left(\chi\left(x_{0}\right)+\varepsilon, t\right)+$ $\gamma_{3}\left(\left\|w_{0+}\right\|_{[0, t)}\right) \forall t \geq 0$ and all $w_{0+} \in \mathcal{W}^{+}$. Since $\varepsilon$ is an arbitrarily chosen positive number, we have that (2.28) holds with $\beta_{2}=\beta_{3}$ and $\gamma_{2}=\gamma_{3}$.

3. Robust stability and main results. Given normed signal spaces $\mathcal{U}, \mathcal{Y}$ and $\mathcal{W} \triangleq \mathcal{U} \times \mathcal{Y}$, consider the closed-loop system $[P, C]$ with the plant $P$ and the controller $C$ (Definition 2.14). Let the perturbed plant $\tilde{P}$ and the perturbed closed-loop system $[\tilde{P}, C]$ be represented by the sets $\mathfrak{B}_{\tilde{P}} \subseteq \mathcal{U}_{e} \times \mathcal{Y}_{e}$ and $\mathfrak{B}_{\tilde{P} / / C} \subseteq \mathcal{W}_{e} \times \mathcal{W}_{e}$, respectively. Let $\mathfrak{S}_{P}, \mathfrak{S}_{\tilde{P}}, \mathfrak{S}_{C}, \mathfrak{S}_{P / / C}$, and $\mathfrak{S}_{\tilde{P} / / C}$ be the corresponding initial state spaces of $\mathfrak{B}_{P}, \mathfrak{B}_{\tilde{P}}, \mathfrak{B}_{C}, \mathfrak{B}_{P / / C}$, and $\mathfrak{B}_{\tilde{P} / / C}$ at initial time 0 , respectively. Note that the graph $\mathcal{G}_{P}^{w_{1-}}, \mathcal{G}_{\tilde{P}}^{\tilde{w}_{1-}}$, and $\mathcal{G}_{C}^{w_{2-}}$ for any $w_{1-} \in \mathfrak{B}_{P}^{-}$, any $\tilde{w}_{1-} \in \mathfrak{B}_{\tilde{P}}^{-}$, and any $w_{2-} \in \mathfrak{B}_{C}^{-}$are similarly defined according to (2.2). 
3.1. General systems. The main result of the paper is given next. It forms a direct extension of [13, Theorems 1 and 6$]$ to include nonzero initial conditions. Before giving the result, we recall that an operator (possibly nonlinear) $\Psi: \mathcal{W}^{+} \rightarrow \mathcal{W}^{+}$is said to be relatively continuous if, for all operators $\Phi: \mathcal{W}^{+} \rightarrow \mathcal{W}^{+}$with $R_{[0, \tau)} \Phi$ compact for any $0<\tau<\infty$, the operator $R_{[0, \tau)}(\Phi \circ \Psi): \mathcal{W}^{+} \rightarrow \mathcal{W}[0, \tau)$ is continuous (see, e.g., [8, p. 1229]).

Assumption 3.1. The following assumptions on the normed vector space $\mathcal{W}^{+}$are only required in the proof of Theorem 3.2 with condition II:

- For any $x \in \mathcal{W}_{e}^{+}$, if $\|x\|<\infty$, then $x \in \mathcal{W}^{+}$.

- The normed vector space $\mathcal{W}^{+}$(not necessarily complete) is truncation complete, i.e., $\mathcal{W}[0, \tau)$ is complete for any $0<\tau<\infty$.

- For any time interval $J \triangleq[0, \tau)$ with $0<\tau<\infty$, there exists a continuous map $E_{J}: \mathcal{W}(J) \rightarrow \mathcal{W}^{+}$such that $R_{J} x=R_{J}\left(E_{J} x\right)$ for any $x \in \mathcal{W}(J)$.

Theorem 3.2. Assume that $P, \tilde{P}$, and $C$ are well-posed and causal systems, that $[P, C]$ is time-invariant, well-posed, and causal, and that $[\tilde{P}, C]$ is causal. Let $[P, C]$ be input to output stable, i.e., there exist functions $\beta \in \mathcal{K} \mathcal{L}$ and $\gamma \in \mathcal{K}_{\infty}$ such that $\forall x_{0}=\left(x_{10}, x_{20}\right) \in \mathfrak{S}_{P} \times \mathfrak{S}_{C}, \forall w_{0+} \in \mathcal{W}^{+}, \forall t>0$,

$$
\left|\left(\overline{\Pi_{P / / C}^{x_{0}}} w_{0+}\right)(t)\right| \leq \beta\left(\chi\left(x_{0}\right), t\right)+\gamma\left(\left\|w_{0+}\right\|_{[0, t)}\right) .
$$

If there exist functions $\sigma_{0}, \sigma \in \mathcal{K}_{\infty}$, and $\beta_{0} \in \mathcal{K} \mathcal{L}$ such that for any $\tilde{w}_{1-} \in \mathcal{W}^{-} \cap \mathfrak{B}_{\tilde{P}}^{-}$ there exists a $w_{1-} \in \mathcal{W}^{-} \cap \mathfrak{B}_{P}^{-}$with

$$
\left\|w_{1-}\right\| \leq \sigma_{0}\left(\left\|\tilde{w}_{1-}\right\|\right)
$$

and a causal surjective operator $\Phi: \operatorname{dom}(\Phi) \subseteq \mathcal{G}_{P}^{w_{1-}} \rightarrow \mathcal{G}_{\tilde{P}}^{\tilde{w}_{1-}}$ satisfying $\forall t>h \geq$ $0, \forall w_{1+} \in \operatorname{dom}(\Phi)$,

$$
\left|\left((\Phi-I) w_{1+}\right)(t)\right| \leq \beta_{0}\left(\left\|w_{1-} \wedge w_{1+}\right\|_{(-\infty, h]}, t-h\right)+\sigma\left(\left\|w_{1+}\right\|_{[h, t)}\right) ;
$$

in addition, if there exist two functions $\rho, \varepsilon$ of class $\mathcal{K}_{\infty}$ such that $\forall s \geq 0$,

$$
\sigma \circ(I+\rho) \circ \gamma(s) \leq(I+\varepsilon)^{-1}(s),
$$

and either of the following conditions is satisfied,

I. $[\tilde{P}, C]$ is well-posed and $\overline{\prod_{\tilde{P} / / C}^{\tilde{x}_{0}}}\left(\mathcal{W}^{+}\right) \subseteq \mathcal{W}^{+}$for any $\tilde{x}_{0} \in \mathfrak{S}_{\tilde{P}} \times \mathfrak{S}_{C}$,

II. Assumption 3.1 holds for $\mathcal{W}^{+},[\tilde{P}, C]$ has the uniqueness property, and $\overline{\Pi_{P / / C}^{x_{0}}}$ is relatively continuous for any $x_{0} \in \mathfrak{S}_{P} \times \mathfrak{S}_{C}$, and $R_{[0, \tau)}(\Phi-I) \forall \tau \in(0, \infty)$ is compact,

then the closed-loop system $[\tilde{P}, C]$ is also input to output stable. More specifically, for any function $\alpha$ of class $\mathcal{K}_{\infty}$, there exists a function $\tilde{\beta} \in \mathcal{K} \mathcal{L}$ such that $\forall \tilde{x}_{0} \in$ $\mathfrak{S}_{\tilde{P}} \times \mathfrak{S}_{C}, \forall \tilde{w}_{0+} \in \mathcal{W}^{+}, \forall t>0$,

$$
\left|\left(\overline{\Pi_{\tilde{P} / / C}^{\tilde{x}_{0}}} \tilde{w}_{0+}\right)(t)\right| \leq \tilde{\beta}\left(\chi\left(\tilde{x}_{0}\right), t\right)+(\alpha+\tilde{\gamma})\left(\left\|\tilde{w}_{0+}\right\|_{[0, t)}\right),
$$

where $\tilde{\gamma} \in \mathcal{K}_{\infty}$ is defined by

$$
\tilde{\gamma}(r) \triangleq(\sigma+I) \circ(I+\rho) \circ \gamma \circ\left(I+\varepsilon^{-1}\right)^{3}(r) \forall r \geq 0 .
$$

Proof. (Part I) For any $\tilde{w}_{0+} \in \mathcal{W}^{+}$and any $\tilde{x}_{0} \in \mathfrak{S}_{\tilde{P}} \times \mathfrak{S}_{C}$, choose any bounded $\left(\tilde{w}_{1-}, w_{2-}\right) \in \tilde{x}_{0}$ and let $\tilde{w}_{0-}=\tilde{w}_{1-}+w_{2-}$. Since $[\tilde{P}, C]$ is well-posed and causal 
and $\overline{\Pi_{\tilde{P} / / C}^{\tilde{x}_{0}}}\left(\mathcal{W}^{+}\right) \subseteq \mathcal{W}^{+}$, there exists a unique $\left(\tilde{w}_{1+}, w_{2+}\right) \in \mathcal{W}^{+} \times \mathcal{W}^{+}$such that $\tilde{w}_{1+} \in \mathcal{G}_{\tilde{P}}^{\tilde{w}_{1-}}, w_{2+} \in \mathcal{G}_{C}^{w_{2-}}$, and $\tilde{w}_{0+}=\tilde{w}_{1+}+w_{2+}$, i.e., the operator $\overline{\Pi_{\tilde{P} / / C}^{\tilde{x}_{0}}}: \mathcal{W}^{+} \rightarrow$ $\mathcal{W}^{+}, \tilde{w}_{0+} \mapsto \tilde{w}_{1+}$ is well-defined and causal.

Under conditions in Theorem 3.2, there exists a $w_{1-} \in \mathcal{W}^{-} \cap \mathfrak{B}_{P}^{-}$for $\tilde{w}_{1-}$ such that $\left\|w_{1-}\right\| \leq \sigma_{0}\left(\left\|\tilde{w}_{1-}\right\|\right)$ (see (3.2)), and thus

$$
\left\|\left(w_{1-}, w_{2-}\right)\right\| \leq\left(\sigma_{0}+I\right)\left(\left\|\left(\tilde{w}_{1-}, w_{2-}\right)\right\|\right) .
$$

In addition, there exists a causal surjective operator $\Phi: \operatorname{dom}(\Phi) \subseteq \mathcal{G}_{P}^{w_{1-}} \rightarrow \mathcal{G}_{\tilde{P}}^{\tilde{w}_{1-}}$. It follows from the surjection of $\Phi$ that there exists $w_{1+} \in \operatorname{dom}(\Phi) \subseteq \mathcal{G}_{P}^{w_{1-}}$ satisfying $\Phi\left(w_{1+}\right)=\tilde{w}_{1+}$. We choose $x_{0} \triangleq\left(\left[w_{1-}\right],\left[w_{2-}\right]\right) \in \mathfrak{S}_{P} \times \mathfrak{S}_{C}$ and let $w_{0-}=w_{1-}+w_{2-}$ and $w_{0+} \triangleq w_{1+}+w_{2+}$. It follows from the well-posedness of $[P, C]$ that $\overline{\Pi_{P / / C}^{x_{0}}}\left(w_{0+}\right)=$ $w_{1+}$, and thus the following equations hold:

$$
\begin{aligned}
\overline{\Pi_{\tilde{P} / / C}^{\tilde{x}_{0}}}\left(\tilde{w}_{0+}\right) & =\tilde{w}_{1+}=\Phi \circ \overline{\Pi_{P / / C}^{x_{0}}}\left(w_{0+}\right), \\
\tilde{w}_{0+} & =\left(I+(\Phi-I) \circ \overline{\Pi_{P / / C}^{x_{0}}}\right)\left(w_{0+}\right) .
\end{aligned}
$$

For ease of notation, we define $w_{i} \triangleq\left(w_{i-} \wedge w_{i+}\right)$ for $i=0,1,2$ and $\tilde{w}_{j} \triangleq\left(\tilde{w}_{j-} \wedge \tilde{w}_{j+}\right)$ for $j=0,1$. From (3.1) and Theorem 2.19 and using the time-invariance and causality of $[P, C]$, we have

$$
\left|w_{1}(t)\right| \leq \beta\left(\left\|\left(w_{1}, w_{2}\right)\right\|_{(-\infty, h]}, t-h\right)+\gamma\left(\left\|w_{0}\right\|_{[h, t]}\right) \forall t \geq h \geq 0 .
$$

Note that, for any function $\mu:[0, r) \rightarrow[0, \infty)$ of class $\mathcal{K}$, any function $\nu$ of class $\mathcal{K}_{\infty}$, and any $a \geq 0, b \geq 0$ with $a+b<r$, we have ${ }^{7}$

$$
\mu(a+b) \leq \mu \circ(I+\nu)(a)+\mu \circ\left(I+\nu^{-1}\right)(b) .
$$

Next, we estimate the upper bound of $\left\|\left(w_{1}, w_{2}\right)\right\|$ by first giving the upper bound of $\left\|w_{0+}\right\|$. It follows from (3.9) that

$$
\begin{aligned}
\left\|w_{0+}\right\| \leq & \left\|\tilde{w}_{0+}\right\|+\left\|(I-\Phi)\left(\overline{\Pi_{P / / C}^{x_{0}}} w_{0+}\right)\right\| \\
\leq & \left\|\tilde{w}_{0+}\right\|+\beta_{0}\left(\left\|w_{1-}\right\|, 0\right)+\sigma\left(\left\|\overline{\Pi_{P / / C}^{x_{0}}} w_{0+}\right\|\right)[\text { by }(3.3)] \\
\leq & \left\|\tilde{w}_{0+}\right\|+\beta_{0}\left(\left\|w_{1-}\right\|, 0\right)+\sigma\left(\beta\left(\left\|\left(w_{1-}, w_{2-}\right)\right\|, 0\right)+\gamma\left(\left\|w_{0+}\right\|\right)\right)[\text { by }(3.10)] \\
\leq & \left\|\tilde{w}_{0+}\right\|+\beta_{0}\left(\left(\sigma_{0}+I\right)\left(\left\|\left(\tilde{w}_{1-}, w_{2-}\right)\right\|\right), 0\right)+\sigma \circ(I+\rho) \circ \gamma\left(\left\|w_{0+}\right\|\right) \\
& +\sigma \circ\left(I+\rho^{-1}\right) \circ \beta\left(\left(\sigma_{0}+I\right)\left(\left\|\left(\tilde{w}_{1-}, w_{2-}\right)\right\|\right), 0\right)[\text { by }(3.7) \text { and }(3.11)] .
\end{aligned}
$$

Since condition (3.4) is satisfied and $\left(I-(I+\varepsilon)^{-1}\right)^{-1}(\cdot)=\left(I+\varepsilon^{-1}\right)(\cdot)$, we see that

$$
\left\|w_{0+}\right\| \leq\left(I+\varepsilon^{-1}\right)\left(\left\|\tilde{w}_{0+}\right\|+\Delta\left(\left\|\left(\tilde{w}_{1-}, w_{2-}\right)\right\|\right)\right),
$$

where function $\Delta \in \mathcal{K}$ is defined by

$$
\Delta(r) \triangleq \beta_{0}\left(\left(\sigma_{0}+I\right)(r), 0\right)+\sigma \circ\left(I+\rho^{-1}\right) \circ \beta\left(\left(\sigma_{0}+I\right)(r), 0\right) \forall r \geq 0 .
$$

\footnotetext{
${ }^{7}$ If $b \leq \nu(a)$, then $\mu(a+b) \leq \mu \circ(I+\nu)(a)$; and if $a \leq \nu^{-1}(b)$, then $\mu(a+b) \leq \mu \circ\left(I+\nu^{-1}\right)(b)$.
} 
Define three functions $\alpha_{i} \in \mathcal{K}_{\infty},(i=1,2,3)$ by

$$
\begin{aligned}
& \alpha_{1}(s) \triangleq\left(\sigma_{0}+I\right)(s)+2 \beta\left(\left(\sigma_{0}+I\right)(s), 0\right) \forall s \geq 0 ; \\
& \alpha_{2}(s) \triangleq \alpha_{1}(s)+(2 \gamma+I) \circ\left(I+\varepsilon^{-1}\right) \circ(I+\varepsilon) \circ \Delta(s) \forall s \geq 0 ; \\
& \alpha_{3}(s) \triangleq(2 \gamma+I) \circ\left(I+\varepsilon^{-1}\right) \circ\left(I+\varepsilon^{-1}\right)(s) \forall s \geq 0 .
\end{aligned}
$$

Thus, we have

$$
\begin{aligned}
\left\|\left(w_{1}, w_{2}\right)\right\| \leq & \left\|\left(w_{1-}, w_{2-}\right)\right\|+2\left\|w_{1+}\right\|+\left\|w_{0+}\right\| \\
\leq & \left(\sigma_{0}+I\right)\left(\left\|\left(\tilde{w}_{1-}, w_{2-}\right)\right\|\right)+2 \beta\left(\left(\sigma_{0}+I\right)\left(\left\|\left(\tilde{w}_{1-}, w_{2-}\right)\right\|\right), 0\right) \\
& +2 \gamma\left(\left\|w_{0+}\right\|\right)+\left\|w_{0+}\right\|[\text { by }(3.7) \text { and }(3.10)] \\
\leq & \alpha_{1}\left(\left\|\left(\tilde{w}_{1-}, w_{2-}\right)\right\|\right)[\text { by }(3.12)] \\
& +(2 \gamma+I) \circ\left(I+\varepsilon^{-1}\right)\left(\left\|\tilde{w}_{0+}\right\|+\Delta\left(\left\|\left(\tilde{w}_{1-}, w_{2-}\right)\right\|\right)\right) \\
\leq & \alpha_{2}\left(\left\|\left(\tilde{w}_{1-}, w_{2-}\right)\right\|\right)+\alpha_{3}\left(\left\|\tilde{w}_{0+}\right\|\right) \triangleq s_{\infty}[\text { by }(3.11)] .
\end{aligned}
$$

By using (3.9), for any $t>0$, we have

$$
\begin{aligned}
\left|w_{0+}(t)\right| \leq & \left|\tilde{w}_{0+}(t)\right|+\left|\left((\Phi-I) \circ \overline{\Pi_{P / / C}^{x_{0}}}\left(w_{0+}\right)\right)(t)\right| \\
\leq & \left\|\tilde{w}_{0}\right\|_{[0, t)}+\beta_{0}\left(\left\|w_{1}\right\|_{(-\infty, t / 2]}, t-t / 2\right)+\sigma\left(\left\|w_{1+}\right\|_{[t / 2, t]}\right)[\text { by }(3.3)] \\
\leq & \left\|\tilde{w}_{0}\right\|_{[0, t)}+\beta_{0}\left(s_{\infty}, t / 2\right)+\sigma\left(\beta\left(s_{\infty}, t / 4\right)+\gamma\left(\left\|w_{0}\right\|_{[t / 4, t)}\right)\right)[\text { by }(3.10)] \\
\leq & \left\|\tilde{w}_{0}\right\|_{[0, t)}+\beta_{0}\left(s_{\infty}, t / 2\right)+\sigma \circ\left(I+\rho^{-1}\right) \circ \beta\left(s_{\infty}, t / 4\right) \\
& +\sigma \circ(I+\rho) \circ \gamma\left(\left\|w_{0}\right\|_{[t / 4, t)}\right)[\text { by }(3.11)] \\
\leq & \left\|\tilde{w}_{0}\right\|_{[0, t)}+\beta_{1}\left(s_{\infty}, t\right)+(I+\varepsilon)^{-1}\left(\left\|w_{0+}\right\|_{[t / 4, t)}\right),
\end{aligned}
$$

where $s_{\infty}$ is defined by (3.14) and $\beta_{1} \in \mathcal{K} \mathcal{L}$ is defined by

$$
\beta_{1}(r, s) \triangleq \beta_{0}(r, s / 2)+\sigma \circ\left(I+\rho^{-1}\right) \circ \beta(r, s / 4) \forall r \geq 0, \forall s \geq 0 .
$$

By applying [17, Lemma A.1] to (3.15) (with $\mu=\frac{1}{4}$ and $\lambda=I+\varepsilon^{-1}$ ), it follows that a function $\beta_{2}$ of class $\mathcal{K} \mathcal{L}$ exists such that $\forall t>0$,

$$
\begin{aligned}
\left|w_{0+}(t)\right| & \leq \beta_{2}\left(s_{\infty}, t\right)+\left(I-(I+\varepsilon)^{-1}\right)^{-1} \circ\left(I+\varepsilon^{-1}\right)\left(\left\|\tilde{w}_{0+}\right\|_{[0, t)}\right) \\
& \leq \beta_{2}\left(s_{\infty}, t\right)+\left(I+\varepsilon^{-1}\right)^{2}\left(\left\|\tilde{w}_{0}\right\|_{[0, t)}\right) .
\end{aligned}
$$

Define functions $\beta_{3} \in \mathcal{K} \mathcal{L}, \hat{\beta} \in \mathcal{K} \mathcal{L}$, and $\alpha_{4} \in \mathcal{K}$ (without loss of generality, we could regard $\alpha_{4}$ as a function of class $\mathcal{K}_{\infty}$ ) as follows $\forall r \geq 0$ and $\forall s \geq 0$ :

$$
\begin{aligned}
\beta_{3}(r, s) & \triangleq \beta_{0}(r, s / 2)+(\sigma+I) \circ\left(I+\rho^{-1}\right) \circ \beta(r, s / 4) ; \\
\beta_{4}(r, s) & \triangleq \beta_{3}(r, s)+(\sigma+I) \circ(I+\rho) \circ \gamma \circ(I+\varepsilon) \circ \beta_{2}(r, s / 4) ; \\
\alpha_{4}(r) & \triangleq \beta_{3}\left(\left(\sigma_{0}+I\right)(r), 0\right)+(\sigma+I) \circ(I+\rho) \circ \gamma \circ\left(I+\varepsilon^{-1}\right) \circ(I+\varepsilon) \circ \Delta(r) .
\end{aligned}
$$

Copyright (c) by SIAM. Unauthorized reproduction of this article is prohibited. 
Hence, by using (3.8), for any $t>0$ we have

$$
\begin{aligned}
\left|\left(\overline{\Pi_{\tilde{P} / / C}^{\tilde{x}_{0}}}\left(\tilde{w}_{0+}\right)\right)(t)\right| \leq & \left\|(\Phi-I) \circ \overline{\Pi_{P / / C}^{x_{0}}}\left(w_{0+}\right)\right\|_{[0, t]}+\left\|\overline{\Pi_{P / / C}^{x_{0}}}\left(w_{0+}\right)\right\|_{[0, t]} \\
\leq & \beta_{0}\left(\left\|w_{1-}\right\|, 0\right)+(\sigma+I)\left(\left\|w_{1+}\right\|_{[0, t]}\right)[\text { by }(3.3)] \\
\leq & \beta_{0}\left(\left\|w_{1-}\right\|, 0\right)+(\sigma+I) \\
& \circ\left(\beta\left(\left\|\left(w_{1-}, w_{2-}\right)\right\|, 0\right)+\gamma\left(\left\|w_{0}\right\|_{[0, t]}\right)\right)[\text { by }(3.10)] \\
\leq & \beta_{3}\left(\left(\sigma_{0}+I\right)\left(\left\|\left(\tilde{w}_{1-}, w_{2-}\right)\right\|\right), 0\right)+(\sigma+I) \circ(I+\rho) \circ \gamma \\
& \circ\left(I+\varepsilon^{-1}\right)\left(\left\|\tilde{w}_{0+}\right\|+\Delta\left(\left\|\left(\tilde{w}_{1-}, w_{2-}\right)\right\|\right)\right)[\text { by }(3.7),(3.12)] \\
\leq & \alpha_{4}\left(\left\|\left(\tilde{w}_{1-}, w_{2-}\right)\right\|\right)+\tilde{\gamma}\left(\left\|\tilde{w}_{0+}\right\|_{[0, t)}\right)
\end{aligned}
$$

with $\tilde{\gamma} \in \mathcal{K}_{\infty}$ defined by (3.6). (Note that $\left(I+\varepsilon^{-1}\right)^{2}(\cdot) \leq\left(I+\varepsilon^{-1}\right)^{3}(\cdot)$.) Moreover,

$$
\begin{aligned}
\left|\left(\overline{\Pi_{\tilde{P} / / C}^{\tilde{x}_{0}}}\left(\tilde{w}_{0+}\right)\right)(t)\right| \leq & \left|\left((\Phi-I) \circ \overline{\Pi_{P / / C}^{x_{0}}}\left(w_{0+}\right)\right)(t)\right|+\left|\left(\overline{\Pi_{P / / C}^{x_{0}}}\left(w_{0+}\right)\right)(t)\right| \\
\leq & \beta_{0}\left(\left\|w_{1}\right\|_{\left(-\infty, \frac{t}{2}\right]}, t-t / 2\right)+(\sigma+I)\left(\left\|w_{1+}\right\|_{\left[\frac{t}{2}, t\right]}\right)[\text { by }(3.3)] \\
\leq & \beta_{0}\left(s_{\infty}, t / 2\right)+(\sigma+I)\left(\beta\left(s_{\infty}, t / 4\right)+\gamma\left(\left\|w_{0}\right\|_{\left[\frac{t}{4}, t\right)}\right)\right)[\text { by }(3.10)] \\
\leq & \beta_{3}\left(s_{\infty}, t\right)+(\sigma+I) \circ(I+\rho) \circ \gamma\left(\left\|w_{0}\right\|_{\left[\frac{t}{4}, t\right)}\right)[\text { by }(3.11)] \\
\leq & \beta_{3}\left(s_{\infty}, t\right)+(\sigma+I) \circ(I+\rho) \circ \gamma \\
& \circ\left(\beta_{2}\left(s_{\infty}, t / 4\right)+\left(I+\varepsilon^{-1}\right)^{2}\left(\left\|\tilde{w}_{0+}\right\|_{[0, t)}\right)\right)[\text { by }(3.17)] \\
\leq & \hat{\beta}\left(s_{\infty}, t\right)+\tilde{\gamma}\left(\left\|\tilde{w}_{0+}\right\|_{[0, t)}\right)
\end{aligned}
$$

with function $\tilde{\gamma} \in \mathcal{K}_{\infty}$ defined by (3.6). Since $s_{\infty}=\alpha_{2}\left(\left\|\left(\tilde{w}_{1-}, w_{2-}\right)\right\|\right)+\alpha_{3}\left(\left\|\tilde{w}_{0+}\right\|\right)$ (see (3.14)), from (3.18) and (3.19) we have for any $t \geq 0$,

$$
\left|\left(\overline{\Pi_{\tilde{P} / / C}^{\tilde{x}_{0}}}\left(\tilde{w}_{0+}\right)\right)(t)\right| \leq \tilde{\gamma}\left(\left\|\tilde{w}_{0+}\right\|\right)+\min \left\{\alpha_{4}\left(\left\|\left(\tilde{w}_{1-}, w_{2-}\right)\right\|\right),\right.
$$

$$
\left.\hat{\beta}\left(\alpha_{2}\left(\left\|\left(\tilde{w}_{1-}, w_{2-}\right)\right\|\right)+\alpha_{3}\left(\left\|\tilde{w}_{0+}\right\|\right), t\right)\right\} .
$$

Given any function $\alpha$ of $\mathcal{K}_{\infty}$, there are only two cases $\left\|\left(\tilde{w}_{1-}, w_{2-}\right)\right\| \leq \alpha_{4}^{-1} \circ \alpha\left(\left\|\tilde{w}_{0+}\right\|\right)$ or $\left\|\tilde{w}_{0+}\right\| \leq \alpha^{-1} \circ \alpha_{4}\left(\left\|\left(\tilde{w}_{1-}, w_{2-}\right)\right\|\right)$, and thus from (3.20) and by considering the fact that for any fixed $t>0$ the function $\hat{\beta}(\cdot, t) \in \mathcal{K}$, we have for any $t \geq 0$,

$$
\begin{aligned}
\left|\left(\overline{\Pi_{\tilde{P} / / C}^{\tilde{x}_{0}}}\left(\tilde{w}_{0+}\right)\right)(t)\right| \leq & \tilde{\gamma}\left(\left\|\tilde{w}_{0+}\right\|\right)+\alpha_{4} \circ \alpha_{4}^{-1} \circ \alpha\left(\left\|\tilde{w}_{0+}\right\|\right) \\
& +\hat{\beta}\left(\alpha_{2}\left(\left\|\left(\tilde{w}_{1-}, w_{2-}\right)\right\|\right)+\alpha_{3} \circ \alpha^{-1} \circ \alpha_{4}\left(\left\|\left(\tilde{w}_{1-}, w_{2-}\right)\right\|\right), t\right) .
\end{aligned}
$$

Since $[\tilde{P}, C]$ is causal, we have, for any $t>0$,

$$
\left|\left(\overline{\Pi_{\tilde{P} / / C}^{\tilde{x}_{0}}} \tilde{w}_{0+}\right)(t)\right| \leq \tilde{\beta}\left(\left\|\left(\tilde{w}_{1-}, w_{2-}\right)\right\|, t\right)+(\alpha+\tilde{\gamma})\left(\left\|\tilde{w}_{0+}\right\|_{[0, t)}\right),
$$

where the function $\tilde{\gamma} \in \mathcal{K}_{\infty}$ is defined by (3.6) and $\tilde{\beta} \in \mathcal{K} \mathcal{L}$ is defined as follows:

$$
\tilde{\beta}(r, t)=\hat{\beta}\left(\alpha_{2}(r)+\alpha_{3} \circ \alpha^{-1} \circ \alpha_{4}(r), t\right) \quad \forall r \geq 0 \forall t \geq 0 .
$$

Since $\tilde{x}_{0}$ and $\tilde{w}_{0+}$ are arbitrarily chosen from $\mathfrak{S}_{\tilde{P}} \times \mathfrak{S}_{C}$ and $\mathcal{W}^{+}$, respectively, we obtain that $[\tilde{P}, C]$ is input to output stable. Moreover, by Theorem 2.19 , for any given function $\alpha \in \mathcal{K}_{\infty}$, from (3.21) we have that (3.5) holds with $\tilde{\beta}$ defined by (3.22). 
(Part II) For any $\tilde{w}_{0+} \in \mathcal{W}^{+}$and any $\tilde{x}_{0} \in \mathfrak{S}_{\tilde{P}} \times \mathfrak{S}_{C}$, choose any bounded $\left(\tilde{w}_{1-}, w_{2-}\right) \in \tilde{x}_{0}$ and let $\tilde{w}_{0-}=\tilde{w}_{1-}+w_{2-}$. Under conditions in Theorem 3.2, there exists a $w_{1-} \in \mathcal{W}^{-} \cap \mathfrak{B}_{P}^{-}$for $\tilde{w}_{1-}$ such that $\left\|w_{1-}\right\| \leq \sigma_{0}\left(\left\|\tilde{w}_{1-}\right\|\right)$ (see (3.2)), and thus inequality (3.7) holds. In addition, there exists a causal surjective operator $\Phi: \operatorname{dom}(\Phi) \subseteq \mathcal{G}_{P}^{w_{1-}} \rightarrow \mathcal{G}_{\tilde{P}}^{\tilde{w}_{1-}}$ such that $R_{J}(\Phi-I)$ is compact with $J \triangleq[0, \tau)$ for any $0<\tau<\infty$. We choose $x_{0} \triangleq\left(\left[w_{1-}\right],\left[w_{2-}\right]\right) \in \mathfrak{S}_{P} \times \mathfrak{S}_{C}$ and let $w_{0-}=w_{1-}+w_{2-}$. Consider the equation

$$
\begin{aligned}
R_{J} \tilde{w}_{0+} & =R_{J}\left(I+(\Phi-I) \circ \overline{\Pi_{P / / C}^{x_{0}}}\right)\left(z_{0+}\right) \\
& =R_{J}\left(I-\overline{\Pi_{P / / C}^{x_{0}}}\right)\left(z_{0+}\right)+R_{J} \Phi \circ \overline{\Pi_{P / / C}^{x_{0}}}\left(z_{0+}\right) .
\end{aligned}
$$

Define a set $M$ as

$$
M=\left\{\bar{z}_{0+} \in \mathcal{W}^{+} \mid\left\|\bar{z}_{0+}\right\|_{J} \leq\left(I+\varepsilon^{-1}\right)\left(\left\|\tilde{w}_{0+}\right\|+\Delta\left(\left\|\left(\tilde{w}_{1-}, w_{2-}\right)\right\|\right)\right)\right\}
$$

with $\Delta \in \mathcal{K}$ defined by (3.13) and consider the operator

$$
Q: M \rightarrow \mathcal{W}(J), \quad \bar{z}_{0+} \mapsto R_{J} \tilde{w}_{0+}+R_{J}(I-\Phi) \circ \overline{\Pi_{P / / C}^{x_{0}}}\left(E_{J} \bar{z}_{0+}\right) .
$$

Theorem 2.19 tells us that (3.1) is equivalent to the following expression:

$$
\left|\overline{\Pi_{P / / C}^{x_{0}}}\left(z_{0+}\right)(t)\right| \leq \beta\left(\left\|\left(w_{1-}, w_{2-}\right)\right\|, t\right)+\gamma\left(\left\|z_{0+}\right\|_{[0, t)}\right) \forall t>0, \forall z_{0+} \in \mathcal{W}^{+} .
$$

From (3.25), we have

$$
\begin{aligned}
\left\|Q\left(\bar{z}_{0+}\right)\right\|_{J} \leq & \left\|R_{J} \tilde{w}_{0+}\right\|_{J}+\left\|R_{J}(I-\Phi) \circ \overline{\Pi_{P / / C}^{x_{0}}}\left(E_{J} \bar{z}_{0+}\right)\right\|_{J} \\
\leq & \left\|\tilde{w}_{0+}\right\|+\beta_{0}\left(\left\|w_{1-}\right\|, 0\right)+\sigma\left(\left\|\bar{\Pi}_{P / / C}^{x_{0}} z_{0+}\right\|\right)[\text { by }(3.3)] \\
\leq & \left\|\tilde{w}_{0+}\right\|+\beta_{0}\left(\left\|w_{1-}\right\|, 0\right)+\sigma \circ\left(\beta\left(\left\|\left(w_{1-}, w_{2-}\right)\right\|, 0\right)\right. \\
& \left.+\gamma\left(\left\|z_{0+}\right\|\right)\right)[\text { by }(3.26)] \\
\leq & \left\|\tilde{w}_{0+}\right\|+\beta_{0}\left(\left(\sigma_{0}+I\right)\left(\left\|\left(\tilde{w}_{1-}, w_{2-}\right)\right\|\right), 0\right)+\sigma \circ(I+\rho) \circ \gamma\left(\left\|z_{0+}\right\|\right) \\
& +\sigma \circ\left(I+\rho^{-1}\right) \circ \beta\left(\left(\sigma_{0}+I\right)\left(\left\|\left(\tilde{w}_{1-}, w_{2-}\right)\right\|\right), 0\right)[\text { by }(3.7) \text { and }(3.11)] \\
\leq & \left\|\tilde{w}_{0+}\right\|+\Delta\left(\left\|\left(\tilde{w}_{1-}, w_{2-}\right)\right\|\right)+(I+\varepsilon)^{-1}\left(\left\|z_{0+}\right\|\right)[\text { by }(3.13) \text { and }(3.4)] \\
\leq & \left(I+(I+\varepsilon)^{-1} \circ\left(I+\varepsilon^{-1}\right)\right)\left(\left\|\tilde{w}_{0+}\right\|+\Delta\left(\left\|\left(\tilde{w}_{1-}, w_{2-}\right)\right\|\right)\right)[\text { by }(3.24)] \\
= & \left(I+\varepsilon^{-1}\right)\left(\left\|\tilde{w}_{0+}\right\|+\Delta\left(\left\|\left(\tilde{w}_{1-}, w_{2-}\right)\right\|\right)\right) .
\end{aligned}
$$

Therefore, $Q(M) \subseteq M \subseteq \mathcal{W}(J)$ with $\mathcal{W}(J)$ being a Banach space. (Note that $\mathcal{W}^{+}$is truncation complete.) Since $R_{J}(\Phi-I)$ is compact and $\overline{\Pi_{P / / C}^{x_{0}}}$ is bounded, it follows that $Q$ is compact. From the relative continuity of $\overline{\Pi_{P / / C}^{x_{0}}}$, we know that $Q$ is also continuous. Thus, by applying the Schauder fixed-point theorem [48] to the operator $Q$ : $M \rightarrow \mathcal{W}(J)$, there exists some $\bar{w}_{0+} \in M \subseteq \mathcal{W}(J)$ such that $\bar{w}_{0+}=Q\left(\bar{w}_{0+}\right) \in \mathcal{W}(J)$. Hence, (3.23) has a solution $z_{0+}=E_{J} \bar{w}_{0+}$. Since $\tilde{w}_{1+}^{J} \triangleq \Phi \circ \overline{\Pi_{P / / C}^{x_{0}}}\left(E_{J} \bar{w}_{0+}\right) \in \mathcal{G}_{\tilde{P}}^{\tilde{w}_{1-}}$ and $w_{2+}^{J} \triangleq\left(I-\overline{\Pi_{P / / C}^{x_{0}}}\right)\left(E_{J} \bar{w}_{0+}\right) \in \mathcal{G}_{C}^{w_{2-}}$, it follows from (3.23) that $R_{J} \tilde{w}_{1+}^{J}+R_{J} w_{2+}^{J}=$ $R_{J} \tilde{w}_{0+}$ and that $\tilde{w}_{1+}^{J}, \tilde{w}_{2+}^{J}$ are bounded independent of $J$. This in turn shows that $[\tilde{P}, C]$ has the existence property up to time $\tau$. (Note that $J \triangleq[0, \tau)$.) Since this holds $\forall 0<\tau<\infty$, and $[\tilde{P}, C]$ is causal and has the uniqueness property, it follows from Corollary 2.6 that $[\tilde{P}, C]$ is well-posed. Since both $\tilde{x}_{0}$ and $\tilde{w}_{0+}$ are arbitrarily chosen from $\mathfrak{S}_{\tilde{P}} \times \mathfrak{S}_{C}$ and $\mathcal{W}^{+}$, respectively, we obtain that $\overline{\Pi_{\tilde{P} / / C}^{\tilde{x}_{0}}}\left(\mathcal{W}^{+}\right) \subseteq$ 
$\mathcal{W}^{+}$for any $\tilde{x}_{0} \in \mathfrak{S}_{\tilde{P}} \times \mathfrak{S}_{C}$. The rest of the proof follows as per the proof of Part I.

The main result, Theorem 3.2, in this paper can be regarded as a generalization of Georgiou and Smith's input-output operator robust stability theorem to accommodate the initial conditions, including an appropriate generalization of the nonlinear gap metric [13]. The idea of looking at the abstract framework for studying the stability of interconnected systems is not new. In the paper [34], the authors established an abstract small-gain theorem in an ISS sense, including applications to purely input/output systems represented by input/output operators defined on the following kind of signal spaces:

$$
L_{0}^{\infty}(S) \triangleq\left\{u \in L^{\infty}(S) \mid u(t)=0 \forall t<t_{0} \text { for some } t_{0} \in \mathbb{R}\right\}
$$

with $S$ being any normed linear space and $L^{\infty}(S)$ consisting of all measurable locally essentially bounded maps from $\mathbb{R}$ to $S$. The IOS concept is still a doubly infinite time axis definition, but it precludes, for example, the uncontrollable stable linear case, since exponential functions do not lie in $L_{0}^{\infty}(S)$. Note that the special representation of systems allows the authors to identify the "state" only with the past input without using the past output; moreover, the well-posedness part of the small-gain theorem was not considered or was considered just as a standing assumption; see [34, section 4.5.2] or $[15]$.

3.1.1. Relation between [13, Theorem 1] and Theorem 3.2. In terms of notation in this paper, [13, Theorem 1] can be expressed as follows.

TheOrem 3.3. Consider the feedback configuration in Figure 1. Assume that $P$, $\tilde{P}, C,[P, C]$, and $[\tilde{P}, C]$ are well-posed and causal systems with $\mathfrak{B}_{P}^{-}=\{0\}, \mathfrak{B}_{\tilde{P}}^{-}=\{0\}$, and $\mathfrak{B}_{C}^{-}=\{0\}$. Let $[P, C]$ be stable, i.e., $\left\|\Pi_{P / / C}^{0}\right\|<\infty$. If there exists a casual surjective map $\Phi_{0}$ from $\mathcal{G}_{P}^{0}$ to $\mathcal{G}_{\tilde{P}}^{0}$ with $\Phi(0)=0$ such that

$$
\left\|\left(\Phi_{0}-I\right)\right\|<\left\|\Pi_{P / / C}^{0}\right\|^{-1},
$$

then $[\tilde{P}, C]$ is stable and $\left\|\Pi_{\tilde{P} / / C}^{0}\right\| \leq\left\|\Pi_{P / / C}^{0}\right\| \frac{1+\left\|\left(\Phi_{0}-I\right)\right\|}{1-\left\|\Pi_{P / / C}^{0}\right\| \cdot\left\|\left(\Phi_{0}-I\right)\right\|}$.

In [13], the plant and controller are assumed to be casual mappings from signal spaces to signal spaces which are only defined on a positive time axis. The properties of mapping zero input to zero output for the plant and controller implicity require that they have zero initial conditions. Thus, we assume that $P, \tilde{P}, C$ are well-posed and causal systems with $\mathfrak{B}_{P}^{-}=\{0\}, \mathfrak{B}_{\tilde{P}}^{-}=\{0\}$, and $\mathfrak{B}_{C}^{-}=\{0\}$ in terms of notation of this paper for the above theorem. That the nominal and perturbed closed-loop systems are casual and well-posed are also standing assumptions in [13]. Also, notice that the condition (3.27) is equivalent to [13, Theorem 1, Condition (2)].

Consider an LTI system $\dot{x}=A x+B u, y=C x+D u, x(0)=x_{0}$ and suppose that $(A, B)$ is stabilizable and $(A, C)$ is detectable. It follows from [35, section 6.3 , Theorem 4] and [16, Example 10.4.1] that the following three statements are equivalent: (1) the matrix $A$ is stable; (2) the LTI system with zero initial conditions is stable with $L^{\infty}$-linear gain, ${ }^{8}$ and (3) the LTI system with initial conditions is input to output stable with $L^{\infty}$-linear gain, ${ }^{9}$ and moreover, the linear gain in (3) can be chosen as the same one in (2) from linearity of the system. In the following, we show to some

\footnotetext{
${ }^{8}$ That is, $\sup \left\{\|y\|_{L^{\infty}[0, t]} /\|u\|_{L^{\infty}[0, t]}: t>0,\|u\|_{L^{\infty}[0, t]} \neq 0, x(0)=0\right\}<\infty$.

${ }^{9}$ That is, $|y(t)| \leq \beta\left(\left|x_{0}\right|, t\right)+\gamma\left(\|u\|_{L^{\infty}[0, t]}\right) \forall t \geq 0$ with $\beta \in \mathcal{K} \mathcal{L}$ and a linear function $\gamma \in \mathcal{K}_{\infty}$.
} 
extent that our robust stability theorem represents a generalization of the inputoutput operator robust stability theorem of Georgiou and Smith, to include the case of initial conditions.

Theorem 3.4. Under the conditions that $P, \tilde{P}, C,[P, C]$, and $[\tilde{P}, C]$ are LTI systems, that $P$ and $\tilde{P}$ are controllable and observable, and that $[P, C]$ and $[\tilde{P}, C]$ are stabilizable and detectable, the first part of Theorem 3.2 (i.e., with condition $\mathrm{I}$ ) is equivalent to Theorem 3.3 (i.e., [13, Theorem 1]).

Proof. We let the premises of the first parts of Theorems 3.2 and 3.3 be denoted by $A_{1}$ and $A_{2}$, and the conclusions of the first parts of Theorems 3.2 and 3.3 be denoted by $B_{1}$ and $B_{2}$, respectively. To establish equivalence we need to show that $\left(A_{1} \Rightarrow B_{1}\right) \Leftrightarrow\left(A_{2} \Rightarrow B_{2}\right)$. Under the conditions in Theorem 3.4, we know that the LTI nominal closed-loop system $[P, C]$ with zero initial conditions is stable with $L^{\infty}$-linear gain if and only if $[P, C]$ with initial conditions is input to output stable with the same $L^{\infty}$-linear gain, i.e., gain function $\gamma$ in (3.1) in Theorem 3.2 is a linear function such that $\gamma(s)=\left\|\Pi_{P / / C}^{0}\right\| \cdot s$ for $s \geq 0$. From section 4.1 below (especially Proposition 4.2), the gap function $\sigma$ in (3.3) in Theorem 3.2 is a linear function such that $\sigma(s)=\left\|\left(\Phi_{0}-I\right)\right\| \cdot s$ for $s \geq 0$. Hence, condition (3.4) is equivalent to $\left\|\Pi_{P / / C}^{0}\right\| \cdot\left\|\left(\Phi_{0}-I\right)\right\|<1$, and so $A_{1} \Leftrightarrow A_{2}$. For the LTI perturbed closed-loop system $[\tilde{P}, C]$, we know that $[\tilde{P}, C]$ with zero initial conditions is stable with $L^{\infty}$-linear gain if and only if $[\tilde{P}, C]$ with initial conditions is input to output stable with the same $L^{\infty}$-linear gain. This implies $B_{1} \Leftrightarrow B_{2}$. Thus, we get $\left(A_{1} \Rightarrow B_{1}\right) \Leftrightarrow\left(A_{2} \Rightarrow B_{2}\right)$. Hence, we know that the first part of Theorem 3.2 is equivalent to Theorem 3.3.

3.2. Finite-time reachable systems. Given normed signal spaces $\mathcal{U}, \mathcal{Y}$, and $\mathcal{W} \triangleq \mathcal{U} \times \mathcal{Y}$, consider the system $Q$ represented by the set $\mathfrak{B}_{Q}$ (see Definition 2.1) and the initial state space $\mathfrak{S}_{Q}$ of $Q$ at initial time 0 defined by Definition 2.7. Let $\delta \in(0, \infty)$, and then the system $Q$ is called finite-time $\delta$-reachable if for any $x_{0} \in \mathfrak{S}_{Q}$ there exists a $w_{-} \in x_{0}$ such that $w_{-}(t)=0 \forall t \in(-\infty,-\delta)$. The system $Q$ is called finite-time reachable if there exist a $\delta \in(0, \infty)$ such that $Q$ is finite-time $\delta$-reachable.

We will now let $t_{0}>0$ be the given initial time and $\mathfrak{S}_{Q}^{t_{0}}$ be the initial state space of $Q$ at time $t_{0}$. Suppose that the system $Q$ is finite-time $t_{0}$-reachable (i.e., for any $x_{0} \in \mathfrak{S}_{Q}^{t_{0}}$ there exists a $w_{-} \in x_{0}$ such that $\left.w_{-}(t)=0 \forall t<0\right)$. Let us define a map $\iota$ as follows:

$$
\iota: x_{0} \mapsto\left\{w \in \mathcal{W}\left[0, t_{0}\right] \mid 0_{(-\infty, 0)} \wedge w \in x_{0}\right\} \quad \forall x_{0} \in \mathfrak{S}_{Q}^{t_{0}} .
$$

Since $Q$ is finite-time $t_{0}$-reachable, we know that $\iota\left(x_{0}\right) \neq \emptyset$ for any $x_{0} \in \mathfrak{S}_{Q}^{t_{0}}$. Denote by $\iota\left(\mathfrak{S}_{Q}^{t_{0}}\right)$ the image of above map $\iota$.

TheOrem 3.5. The map $\iota: \mathfrak{S}_{Q}^{t_{0}} \rightarrow \iota\left(\mathfrak{S}_{Q}^{t_{0}}\right)$ is a bijection.

Proof. We only need to prove that $\iota$ is an injection. To this end, we have to show $x_{1}=x_{2}$ for any $x_{1}, x_{2} \in \mathfrak{S}_{Q}^{t_{0}}$ satisfying $\iota\left(x_{1}\right)=\iota\left(x_{2}\right)$. Choose any $w \in \iota\left(x_{1}\right)=\iota\left(x_{2}\right)$, from (3.28) we know that $0_{(-\infty, 0)} \wedge w$ belongs to both $x_{1}$ and $x_{2}$. Thus, from the definition of initial state space $\mathfrak{S}_{Q}^{t_{0}}$, we get $x_{1}=x_{2}$.

Recalling the definition of a graph of a system for past trajectory 0 (see (2.2)), i.e., $\mathcal{G}_{Q}^{0} \triangleq\left\{w_{+} \in \mathcal{W}^{+} \mid 0_{(-\infty, 0)} \wedge w_{+} \in \mathfrak{B}_{Q}\right\}$, Theorem 3.6 shows that the image of the map $\iota$ produces a partition for the restriction of graph $\mathcal{G}_{Q}^{0}$ to $\left[0, t_{0}\right]$.

Theorem 3.6. The image $\iota\left(\mathfrak{S}_{Q}^{t_{0}}\right)$ of the map $\iota$ is a partition of $\left.\mathcal{G}_{Q}^{0}\right|_{\left[0, t_{0}\right]}$.

Proof. Since $Q$ is finite-time $t_{0}$-reachable, we have $\iota\left(x_{0}\right) \neq \emptyset$ for any $x_{0} \in \mathfrak{S}_{Q}^{t_{0}}$ and thus $\emptyset \notin \iota\left(\mathfrak{S}_{Q}^{t_{0}}\right)$. For any $\left.w_{\left[0, t_{0}\right]} \in \mathcal{G}_{Q}^{0}\right|_{\left[0, t_{0}\right]}$, there must exist a $x_{0} \in \mathfrak{S}_{Q}^{t_{0}}$ such 
that $0_{(-\infty, 0)} \wedge w_{\left[0, t_{0}\right]} \in x_{0}$, and therefore $w_{\left[0, t_{0}\right]} \in \iota\left(x_{0}\right)$. This together with $\iota\left(\mathfrak{S}_{Q}^{t_{0}}\right) \subseteq$ $\left.\mathcal{G}_{Q}^{0}\right|_{\left[0, t_{0}\right]}$ shows that $\bigcup \iota\left(\mathfrak{S}_{Q}^{t_{0}}\right)=\left.\mathcal{G}_{Q}^{0}\right|_{\left[0, t_{0}\right]}$. For any $x_{1}, x_{2} \in \mathfrak{S}_{Q}^{t_{0}}$ with $\iota\left(x_{1}\right) \neq \iota\left(x_{2}\right)$ (i.e., $x_{1} \neq x_{2}$ by Theorem 3.5), we have $\iota\left(x_{1}\right) \cap \iota\left(x_{2}\right)=\emptyset$ since any common element belongs to both $\iota\left(x_{1}\right)$ and $\iota\left(x_{2}\right)$ will imply $x_{1}=x_{2}$. The above claims show that $\iota\left(\mathfrak{S}_{Q}^{t_{0}}\right)$ is a partition of $\left.\mathcal{G}_{Q}^{0}\right|_{\left[0, t_{0}\right]}$.

By definition of the map $\iota$ (see (3.28)) and Theorem 3.5, we know that, given initial time $t_{0}>0$, for finite-time $t_{0}$-reachable system, we can actually only use trajectories with zero past up to time 0 to define all states at initial time $t_{0}>0$. In this case, we can slightly change the definition of the size (see (2.6)) of any state $x_{t_{0}} \in \mathfrak{S}_{Q}^{t_{0}}$ by another real-valued function $\tilde{\chi}$ :

$$
\tilde{\chi}: \mathfrak{S}_{Q}^{t_{0}} \rightarrow[0, \infty), \quad x_{t_{0}} \mapsto \tilde{\chi}\left(x_{t_{0}}\right) \triangleq \inf _{w \in x_{t_{0}}, w(t)=0(\forall t<0)}\left\{\|w\|_{\left(-\infty, t_{0}\right]}\right\} .
$$

It is easy to see that $\tilde{\chi}\left(x_{t_{0}}\right)=\inf _{w \in \iota\left(x_{t_{0}}\right)}\left\{\|w\|_{\left[0, t_{0}\right]}\right\} \geq \chi\left(x_{t_{0}}\right)$ for any $x_{t_{0}} \in \mathfrak{S}_{Q}^{t_{0}}$. According to above discussions for finite-time reachable systems, by using a new size function (3.29) for initial states and the same procedure of proof for the main Theorem 3.2, we can obtain the following applicable robust stability theorem.

Theorem 3.7. Give initial time $t_{0}>0$ and assume that $P, \tilde{P}$, and $C$ are well-posed, finite-time $t_{0}$-reachable, and causal systems that $[P, C]$ is time-invariant, well-posed, and causal and that $[\tilde{P}, C]$ is causal. Let $[P, C]$ be input to output stable, i.e., there exist functions $\beta \in \mathcal{K} \mathcal{L}$ and $\gamma \in \mathcal{K}_{\infty}$ such that $\forall x_{t_{0}}=\left(x_{1 t_{0}}, x_{2 t_{0}}\right) \in$ $\mathfrak{S}_{P}^{t_{0}} \times \mathfrak{S}_{C}^{t_{0}}, \forall w_{0+} \in \mathcal{W}\left[t_{0}, \infty\right), \forall t>t_{0}$, we have $\left|\left(\overline{\Pi_{P / / C}^{x_{t_{0}}}} w_{0+}\right)(t)\right| \leq \beta\left(\tilde{\chi}\left(x_{t_{0}}\right), t-t_{0}\right)+$ $\gamma\left(\left\|w_{0+}\right\|_{\left[t_{0}, t\right)}\right)$. If there exists a causal surjective mapping $\Phi: \operatorname{dom}(\Phi) \subseteq \mathcal{G}_{P}^{0} \rightarrow \mathcal{G}_{\tilde{P}}^{0}$ and functions $\beta_{0} \in \mathcal{K} \mathcal{L}, \sigma \in \mathcal{K}_{\infty}, \sigma_{0} \in \mathcal{K}_{\infty}$, such that $\|w\|_{\left[0, t_{0}\right]} \leq \sigma_{0}\left(\|\Phi w\|_{\left[0, t_{0}\right]}\right)$ and $|((\Phi-I) w)(t)| \leq \beta_{0}\left(\|w\|_{[0, h]}, t-h\right)+\sigma\left(\|w\|_{[h, t)}\right)$ for any $w \in \operatorname{dom}(\Phi) \subseteq \mathcal{G}_{P}^{0}$ and any $t>h \geq 0$; in addition, if there exist two functions $\rho, \varepsilon$ of class $\mathcal{K}_{\infty}$ such that $\sigma \circ(I+\rho) \circ \gamma(s) \leq(I+\varepsilon)^{-1}(s) \forall s \geq 0$, and either of the following is satisfied,

I. $[\tilde{P}, C]$ is well-posed and $\overline{\Pi_{\tilde{P} / / C}^{\tilde{x}_{0}}}\left(\mathcal{W}\left[t_{0}, \infty\right)\right) \subseteq \mathcal{W}\left[t_{0}, \infty\right)$ for any $\tilde{x}_{t_{0}} \in \mathfrak{S}_{\tilde{P}}^{t_{0}} \times$ $\mathfrak{S}_{C}^{t_{0}}$,

II. Assumption 3.1 holds for $\mathcal{W}\left[t_{0}, \infty\right),[\tilde{P}, C]$ has the uniqueness property, $\overline{\Pi_{P / / C}^{x_{t_{0}}}}$ is relatively continuous for any $x_{t_{0}} \in \mathfrak{S}_{P}^{t_{0}} \times \mathfrak{S}_{C}^{t_{0}}$, and $R_{\left[t_{0}, \tau\right)}(\Phi-I) \forall t_{0}<\tau<$ $\infty$ is compact,

then the closed-loop system $[\tilde{P}, C]$ is also input to output stable. More specifically, for any function $\alpha$ of class $\mathcal{K}_{\infty}$, there exists a function $\tilde{\beta} \in \mathcal{K} \mathcal{L}$ such that, $\forall \tilde{x}_{t_{0}} \in$ $\mathfrak{S}_{\tilde{P}}^{t_{0}} \times \mathfrak{S}_{C}^{t_{0}}, \forall \tilde{w}_{0+} \in \mathcal{W}\left[t_{0}, \infty\right), \forall t>t_{0}$, we have $\left|\left(\overline{\Pi_{\tilde{P} / / C}^{\tilde{x}_{t_{0}}}} \tilde{w}_{0+}\right)(t)\right| \leq \tilde{\beta}\left(\tilde{\chi}\left(\tilde{x}_{t_{0}}\right), t\right)+(\alpha+$ $\tilde{\gamma})\left(\left\|\tilde{w}_{0+}\right\|_{\left[t_{0}, t\right)}\right)$, where $\tilde{\gamma} \in \mathcal{K}_{\infty}$ is defined by $\tilde{\gamma}(\cdot) \triangleq(\sigma+I) \circ(I+\rho) \circ \gamma \circ\left(I+\varepsilon^{-1}\right)^{3}(\cdot)$.

Proof. This follows directly from Theorems 3.2, 3.5, and 3.6.

\section{Applications.}

4.1. Linear time-invariant systems. Let $\mathcal{U} \triangleq L^{\infty}\left(\mathbb{R} ; \mathbb{R}^{m}\right), \mathcal{Y} \triangleq L^{\infty}\left(\mathbb{R} ; \mathbb{R}^{p}\right)$, and $\mathcal{W} \triangleq \mathcal{U} \times \mathcal{Y}$. Suppose that $A, B, C, D$ are real matrices of dimensions $n \times n$, $n \times m, n \times p, m \times p$, respectively, with $(A, B)$ controllable and $(A, C)$ observable. The nominal plant $P$ is defined by the set $\mathfrak{B}_{P} \triangleq \mathfrak{B}_{A, B, C, D}$ with

$$
\mathfrak{B}_{A, B, C, D} \triangleq\left\{\begin{array}{l|l}
(u, y) \in \mathcal{W}_{e} \mid \begin{array}{c}
\dot{x}=A x+B u, y=C x+D u \\
\text { satisfies for some } x \in L_{e}^{\infty}\left(\mathbb{R} ; \mathbb{R}^{n}\right)
\end{array}
\end{array}\right\} .
$$

Copyright $@$ ㅇ by SIAM. Unauthorized reproduction of this article is prohibited. 
Similarly, suppose that $\tilde{A}, \tilde{B}, \tilde{C}, \tilde{D}$ are real matrices of dimensions $\tilde{n} \times \tilde{n}, \tilde{n} \times m$, $\tilde{n} \times p, m \times p$, respectively, with $(\tilde{A}, \tilde{B})$ controllable and $(\tilde{A}, \tilde{C})$ observable. We define

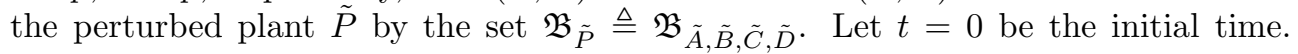
Corresponding to the nominal plant $P$, we define operators

$$
\begin{gathered}
\left(\begin{array}{c}
\mathbb{M} \\
\mathbb{N}
\end{array}\right): \mathcal{U} \rightarrow \mathcal{W}, \quad v \mapsto\left(t \mapsto \int_{-\infty}^{t}\left(\begin{array}{c}
M \\
N
\end{array}\right)(t-\tau) v(\tau) d \tau, t \in \mathbb{R}\right), \\
\mathbb{L}: \mathcal{W} \rightarrow \mathcal{U}, \quad w \mapsto\left(t \mapsto \int_{-\infty}^{t} L(t-\tau) w(\tau) d \tau, t \in \mathbb{R}\right),
\end{gathered}
$$

where the following $\delta$ denotes the unit delta distribution and for any $t \geq 0$,

$$
\begin{aligned}
& \left(\begin{array}{c}
M \\
N
\end{array}\right)(t) \triangleq\left(\begin{array}{c}
F \exp \{t(A+B F)\} B+\delta(t) I_{m \times m} \\
(C+D F) \exp \{t(A+B F)\} B+\delta(t) D
\end{array}\right),
\end{aligned}
$$

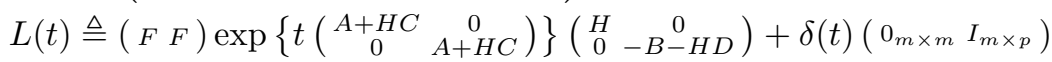

with real matrices $F$ and $H$ chosen such that both $A+B F$ and $A+H C$ are stable (all eigenvalues in $\operatorname{Re} s<0$ ).

From [6, section 4.1] and [35, section 6.4.1], for any $v \in \mathcal{U}$ with $v(t) \equiv 0 \forall t \leq 0$, we have $\mathbb{L}\left(\left(\begin{array}{c}\mathbb{M} \\ \mathbb{N}\end{array}\right) v\right)=v$ and $\left\|\left(\begin{array}{c}\mathbb{M} \\ \mathbb{N}\end{array}\right) v\right\|_{[0, t]} \leq\left\|\left(\begin{array}{c}M \\ N\end{array}\right)\right\|_{A} \cdot\|v\|_{[0, t]}$; and for any $w \in \mathcal{W}$ with $w(t) \equiv 0 \forall t \leq 0$, we have $\|\mathbb{L} w\|_{[0, t]} \leq\|L\|_{A} \cdot\|w\|_{[0, t]}$, where $\|\cdot\|_{A}$ is the norm for distribution. The operators $\left(\begin{array}{c}\tilde{\mathbb{N}} \\ \tilde{\mathbb{N}}\end{array}\right)$ and $\tilde{\mathbb{L}}$ are similarly defined for the perturbed plant $\tilde{P}$.

Proposition 4.1. For the perturbed plant $\tilde{P}$, define a functional $\pi_{1}$ as follows

$$
\pi_{1}: \mathcal{U}^{-} \rightarrow \mathcal{W}^{-} \cap \mathfrak{B}_{\tilde{P}}^{-},\left.\quad u \mapsto\left(\begin{array}{c}
\tilde{\mathbb{M}}(u \wedge 0) \\
\tilde{\mathbb{N}}(u \wedge 0)
\end{array}\right)\right|_{(-\infty, 0]} .
$$

Then, there exists a functional $\pi_{2}: \mathcal{W}^{-} \cap \mathfrak{B}_{\tilde{P}}^{-} \rightarrow\left(\mathcal{U}^{-}\right)_{0}$ and a nonnegative number $\tilde{\rho} \geq 0$ such that for any $\tilde{w}_{-} \in \mathcal{W}^{-} \cap \mathfrak{B}_{\tilde{P}}^{-}$,

$$
\pi_{1} \circ \pi_{2}\left(\tilde{w}_{-}\right)=\left.\left(\begin{array}{l}
\tilde{\mathbb{M}}\left(\pi_{2}\left(\tilde{w}_{-}\right) \wedge 0\right) \\
\tilde{\mathbb{N}}\left(\pi_{2}\left(\tilde{w}_{-}\right) \wedge 0\right)
\end{array}\right)\right|_{(-\infty, 0]}, \quad\left\|\pi_{1} \circ \pi_{2}\left(\tilde{w}_{-}\right)\right\| \leq \tilde{\rho} \cdot\left\|\tilde{w}_{-}\right\|,
$$

and for any $\tilde{w}_{-} \in \mathcal{W}^{-} \cap \mathfrak{B}_{\tilde{P}}^{-}$, the graph $\mathcal{G}_{\tilde{P}}^{\tilde{w}_{-}}$defined by (2.2) satisfies

$$
\mathcal{G}_{\tilde{P}}^{\tilde{w}-}=\left\{\left.\left(\begin{array}{c}
\tilde{\mathbb{M}}\left(\pi_{2}\left(\tilde{w}_{-}\right) \wedge v\right) \\
\tilde{\mathbb{N}}\left(\pi_{2}\left(\tilde{w}_{-}\right) \wedge v\right)
\end{array}\right)\right|_{[0, \infty)} \in \mathcal{W}^{+} \mid v \in \mathcal{U}^{+}\right\}=\mathcal{G}_{\tilde{P}}^{\pi_{1} \circ \pi_{2}\left(\tilde{w}_{-}\right)}
$$

where $\left(\mathcal{U}^{-}\right)_{0} \triangleq\left\{u \in \underset{\tilde{A}}{\mathcal{U}_{\tilde{C}}^{-}} \mid \exists T_{u} \in[0, \infty)\right.$, such that $\left.u(t) \equiv 0 \forall t \leq-T_{u}\right\}$.

Proof. Since $(\tilde{A}, \tilde{C})$ is observable, we have that for any $\tilde{w}_{-} \in \mathcal{W}^{-} \cap \mathfrak{B}_{\tilde{P}}^{-}$, there exists a unique $\tilde{x}_{0} \in \mathbb{R}^{\tilde{n}}$ such that the equations $\dot{\tilde{x}}=\tilde{A} \tilde{x}+\tilde{B} u$ and $y=\tilde{C} \tilde{x}+\tilde{D} u$ hold with $(u(t), y(t))=\tilde{w}_{-}(t)$ for $t \leq 0$ and $\tilde{x}(0)=\tilde{x}_{0}$. In addition, $\left|\tilde{x}_{0}\right| \leq r_{1}\left\|\tilde{w}_{-}\right\|$with $r_{1} \geq 0$ independent of $\tilde{w}_{-}$. Since $(\tilde{A}, \tilde{B})$ is controllable, we obtain that $(\tilde{A}+\tilde{B} \tilde{F}, \tilde{B})$ is controllable, and thus for this $\tilde{x}_{0} \in \mathbb{R}^{\tilde{n}}$, there exists a $v_{\tilde{x}_{0}} \in\left(\mathcal{U}^{-}\right)_{0}$ such that

$$
\tilde{x}_{0}=\int_{-\infty}^{0} \exp \{(0-\tau)(\tilde{A}+\tilde{B} \tilde{F})\} \tilde{B} v_{\tilde{x}_{0}}(\tau) d \tau .
$$

Moreover, $\left\|v_{\tilde{x}_{0}}\right\| \leq r_{2}\left|\tilde{x}_{0}\right|$ with $r_{2} \geq 0$ independent of $\tilde{x}_{0}$. Thus, a functional $\pi_{2}$ can be defined by

$$
\pi_{2}: \mathcal{W}^{-} \cap \mathfrak{B}_{\tilde{P}}^{-} \rightarrow\left(\mathcal{U}^{-}\right)_{0}, \quad \tilde{w}_{-} \mapsto v_{\tilde{x}_{0}}
$$

Copyright $@$ by SIAM. Unauthorized reproduction of this article is prohibited. 
and we have $\left\|\pi_{2}\left(\tilde{w}_{-}\right)\right\| \leq r_{2} r_{1}\left\|\tilde{w}_{-}\right\|$. From similar techniques as in [9, section 4.4], we know that the graph $\mathcal{G}_{\tilde{P}}^{\tilde{w}_{-}}$defined by $(2.2)$ can be expressed as

$$
\mathcal{G}_{\tilde{P}}^{\tilde{w}_{-}}=\left\{\left.\left(\begin{array}{c}
\tilde{\mathbb{M}}_{+} v+\tilde{F} \exp \left\{\cdot \tilde{A}_{\tilde{F}}\right\} \tilde{x}_{0} \\
\tilde{\mathbb{N}}+v+\tilde{C}_{\tilde{F}} \exp \left\{\cdot \tilde{A}_{\tilde{F}}\right\} \tilde{x}_{0}
\end{array}\right)\right|_{[0, \infty)} \in \mathcal{W}^{+} \mid v \in \mathcal{U}^{+}\right\} .
$$

By using (4.6) and (4.7), we know that the right-hand side of (4.8) equals

$$
\left\{\left.\left(\begin{array}{c}
\tilde{\mathbb{M}}\left(\pi_{2}\left(\tilde{w}_{-}\right) \wedge v\right) \\
\tilde{\mathbb{N}}\left(\pi_{2}\left(\tilde{w}_{-}\right) \wedge v\right)
\end{array}\right)\right|_{[0, \infty)} \in \mathcal{W}^{+} \mid v \in \mathcal{U}^{+}\right\} .
$$

From (4.3) and (4.7), we have that (4.4) holds with $\tilde{\rho} \triangleq\left\|\left(\begin{array}{c}\tilde{M} \\ \tilde{N}\end{array}\right)\right\|_{A} \cdot r_{2} \cdot r_{1} \geq 0$, and thus $\mathcal{G}_{\tilde{P}}^{\pi_{1} \circ \pi_{2}\left(\tilde{w}_{-}\right)}$equals (4.9); this implies (4.5).

Proposition 4.2. For any $\tilde{w}_{-} \in \mathcal{W}^{-} \cap \mathfrak{B}_{\tilde{P}}^{-}$, there exists a $w_{-} \in \mathcal{W}^{-} \cap \mathfrak{B}_{P}^{-}$with

$$
\left\|w_{-}\right\| \leq \tilde{\rho} \cdot\left\|\left(\begin{array}{c}
M \\
N
\end{array}\right)\right\|_{A} \cdot\|\tilde{L}\|_{A} \cdot\left\|\tilde{w}_{-}\right\|,
$$

and a causal surjective map $\Phi_{\tilde{w}_{-}}: \mathcal{G}_{P}^{w_{-}} \rightarrow \mathcal{G}_{\tilde{P}}^{\tilde{w}_{-}}$satisfying $\forall t>h \geq 0, \forall w_{+} \in \mathcal{G}_{P}^{w_{-}}$,

$$
\begin{aligned}
\left|\left(\left(\Phi_{\tilde{w}_{-}}-I\right) w_{+}\right)(t)\right| \leq & \beta_{0}\left(\left\|w_{-} \wedge w_{+}\right\|_{(-\infty, h]}, t-h\right) \\
& +\left\|\left(\begin{array}{c}
\tilde{\tilde{N}}-M \\
\tilde{N}-N
\end{array}\right)\right\|_{A} \cdot\|L\|_{A} \cdot\left\|w_{+}\right\|_{[h, t)},
\end{aligned}
$$

where function $\beta_{0} \in \mathcal{K} \mathcal{L}$ and $\tilde{\rho} \geq 0$ is the same as in Proposition 4.1.

Proof. Let the functional $\pi_{1}, \pi_{2}$ be defined as in Proposition 4.1. For any $\tilde{w}_{-} \in$ $\mathcal{W}^{-} \cap \mathfrak{B}_{\tilde{P}}^{-}$, we have that (4.4) and (4.5) hold. It is easy to see that

$$
\left.w_{-} \triangleq\left(\begin{array}{c}
\mathbb{M}\left(\pi_{2}\left(\tilde{w}_{-}\right) \wedge 0\right) \\
\mathbb{N}\left(\pi_{2}\left(\tilde{w}_{-}\right) \wedge 0\right)
\end{array}\right)\right|_{(-\infty, 0]} \in \mathcal{W}^{-} \cap \mathfrak{B}_{P}^{-}
$$

and that the graph $\mathcal{G}_{P}^{w_{-}}$of the nominal plant $P$ is

$$
\mathcal{G}_{P}^{w_{-}}=\left\{\left.\left(\begin{array}{c}
\mathbb{M}\left(\pi_{2}\left(\tilde{w}_{-}\right) \wedge v\right) \\
\mathbb{N}\left(\pi_{2}\left(\tilde{w}_{-}\right) \wedge v\right)
\end{array}\right)\right|_{[0, \infty)} \in \mathcal{W}^{+} \mid v \in \mathcal{U}^{+}\right\} .
$$

Thus, a natural causal surjective map $\Phi_{\tilde{w}_{-}}: \mathcal{G}_{P}^{w_{-}} \rightarrow \mathcal{G}_{\tilde{P}}^{\tilde{w}_{-}}$can be defined as

$$
\left.\left.\left(\begin{array}{l}
\mathbb{M}\left(\pi_{2}\left(\tilde{w}_{-}\right) \wedge v\right) \\
\mathbb{N}\left(\pi_{2}\left(\tilde{w}_{-}\right) \wedge v\right)
\end{array}\right)\right|_{[0, \infty)} \mapsto\left(\begin{array}{c}
\tilde{\mathbb{M}}\left(\pi_{2}\left(\tilde{w}_{-}\right) \wedge v\right) \\
\tilde{\mathbb{N}}\left(\pi_{2}\left(\tilde{w}_{-}\right) \wedge v\right)
\end{array}\right)\right|_{[0, \infty)} \quad \forall v \in \mathcal{U}^{+} .
$$

Since $\left.\pi_{2}\left(\tilde{w}_{-}\right) \in \mathcal{U}^{-}\right)_{0}$, there exists a $T_{\tilde{w}_{-}} \in[0, \infty)$ such that $\pi_{2}\left(\tilde{w}_{-}\right)(t) \equiv 0 \forall t \leq$ $-T_{\tilde{w}_{-}}$. It is elementary to show that

$$
\left\|w_{-}\right\|=\left\|\left(\begin{array}{c}
\mathbb{M}\left(\pi_{2}\left(\tilde{w}_{-}\right) \wedge 0\right) \\
\mathbb{N}\left(\pi_{2}\left(\tilde{w}_{-}\right) \wedge 0\right)
\end{array}\right)\right\|_{\left(-T_{\tilde{w}_{-}}, 0\right]} \leq\left\|\left(\begin{array}{c}
M \\
N
\end{array}\right)\right\|_{A} \cdot\|\tilde{L}\|_{A} \cdot\left\|\left(\begin{array}{c}
\tilde{\mathbb{N}}\left(\pi_{2}\left(\tilde{w}_{-}\right) \wedge 0\right) \\
\tilde{\mathbb{N}}\left(\pi_{2}\left(\tilde{w}_{-}\right) \wedge 0\right)
\end{array}\right)\right\|_{\left[-T_{\left.\tilde{w}_{-}, 0\right]},\right.},
$$

and thus from (4.4), we have that (4.10) holds.

For any $w_{+} \in \mathcal{G}_{P}^{w_{-}}$, there exists a $v \in \mathcal{U}^{+}$such that $w_{+}=\left.\left(\begin{array}{l}\mathbb{M}\left(\pi_{2}\left(\tilde{w}_{-}\right) \wedge v\right) \\ \mathbb{N}\left(\pi_{2}\left(\tilde{w}_{-}\right) \wedge v\right)\end{array}\right)\right|_{[0, \infty)}$. From $(4.12)$, we get $\left(\left(\Phi_{\tilde{w}_{-}}-I\right) w_{+}\right)(t)=\left(\begin{array}{c}\tilde{\mathbb{N}}-\mathbb{M} \\ \tilde{\mathbb{N}}-\mathbb{N}\end{array}\right)\left(\pi_{2}\left(\tilde{w}_{-}\right) \wedge v\right)(t) \forall t \geq 0$; and since $\pi_{2}\left(\tilde{w}_{-}\right)(t) \equiv 0 \forall t \leq-T_{\tilde{w}_{-}}$, we have for any $t \geq h>0$ that

$$
\begin{aligned}
\left|\left(\left(\Phi_{\tilde{w}_{-}}-I\right) w_{+}\right)(t)\right| \leq & \beta_{0}\left(\left\|\left(\begin{array}{c}
\mathbb{M} \\
\mathbb{N}
\end{array}\right)\left(\pi_{2}\left(\tilde{w}_{-}\right) \wedge v\right)\right\|_{\left[-T_{\tilde{w}_{-}}, h\right]}, t-h\right) \\
& +\left\|\left(\begin{array}{c}
\tilde{M}-M \\
\tilde{N}-N
\end{array}\right)\right\|_{A} \cdot\|L\|_{A} \cdot\left\|\left(\begin{array}{c}
\mathbb{M} \\
\mathbb{N}
\end{array}\right)\left(\pi_{2}\left(\tilde{w}_{-}\right) \wedge v\right)\right\|_{[h, t-h)},
\end{aligned}
$$

where $\beta_{0}$ is any function of $\mathcal{K} \mathcal{L}$ such that $\left|\left(\begin{array}{c}\tilde{\mathbb{M}}-\mathbb{M} \\ \tilde{\mathbb{N}}-\mathbb{N}\end{array}\right)(\mathbb{L} v)\left(t_{1}\right)\right| \leq \beta_{0}\left(\|v\|_{\left[0, h_{1}\right]}, t_{1}-\right.$ $\left.h_{1}\right) \forall t_{1} \geq h_{1}$ for any $h_{1} \geq 0$ and any $v \in \mathcal{U}$ with $v(s) \equiv 0 \forall s \in(-\infty, 0] \cup\left[h_{1}, \infty\right)$. Therefore, from $w_{-} \wedge w_{+}=\left(\pi_{2}\left(\tilde{w}_{-}\right) \wedge v\right)$, we obtain that (4.11) holds.

Copyright $@$ by SIAM. Unauthorized reproduction of this article is prohibited. 


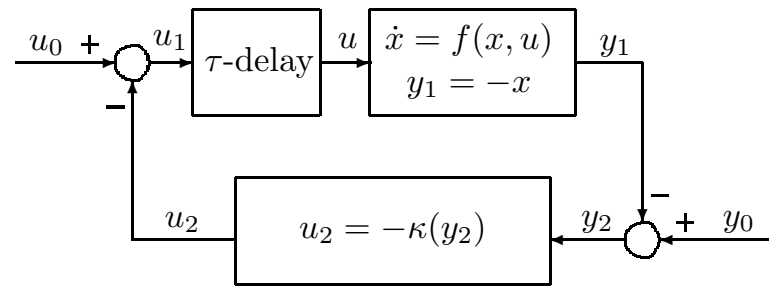

FIG. 2. Nonlinear plant with input delay in closed-loop system.

4.2. General nonlinear plant with input delay. Consider the following closed-loop system, which consists of a nonlinear plant with input delay and a nonlinear controller shown in Figure 2. Assume that both functions $f$ and $\kappa$ are continuous with $f(0,0)=\kappa(0)=0$, that the system $\dot{x}=f(x, u)$ is forward complete (see Definition 2.9), and that the system $\dot{x}=f\left(x, u_{0}+\kappa\left(x+y_{0}\right)\right)$ with input $w_{0}=\left(u_{0}, y_{0}\right)$ and state $x$ is input to state stable [27].

Since both $\kappa$ and $f$ are continuous, there exist $\rho_{1} \in \mathcal{K}_{\infty}$ and $\rho_{2} \in \mathcal{K}_{\infty}$ such that

$$
\kappa(x) \leq \rho_{1}(|x|), \quad|f(x, u)| \leq \rho_{2}(\max \{|x|,|u|\}) .
$$

The nominal closed-loop system (i.e., the closed-loop system shown in Figure 2 for nonlinear plant without input delay) is given by

$$
\begin{aligned}
\dot{x} & =f\left(x, u_{0}+\kappa\left(x+y_{0}\right)\right), \\
u_{1} & =u_{0}+\kappa\left(x+y_{0}\right), \quad y_{1}=-x,
\end{aligned}
$$

and is input to output stable [33], i.e.,

$$
\left|w_{1}(t)\right| \leq \beta\left(\left|x_{0}\right|, t\right)+\gamma\left(\left\|w_{0}\right\|_{[0, t]}\right) \quad \forall t \geq 0, \forall w_{0}, \forall x(0)=x_{0},
$$

for functions $\beta \in \mathcal{K} \mathcal{L}$ and $\gamma \in \mathcal{K}_{\infty}$ with $w_{i} \triangleq\left(u_{i}, y_{i}\right)$ for $i=0,1$.

We consider the determination of input delays which can be tolerated while preserving the input-to-output stability of the closed-loop system shown in Figure 2. To apply Theorem 3.2, we need to measure the distance between the nominal plant and the perturbed plant with input delay. For the convenience of notation, let the nominal plant $P$ and perturbed plant $\tilde{P}$ be defined by the following sets $\mathfrak{B}_{P}$ and $\mathfrak{B}_{\tilde{P}}$, respectively:

$$
\begin{gathered}
\mathfrak{B}_{P}=\left\{w_{1} \in \mathcal{W}_{e} \mid w_{1}=\left(u_{1}, y_{1}\right) \text { satisfies }(4.16) \text { for some } x\right\}, \\
\dot{x}=f\left(x, u_{1}\right), \quad y_{1}=-x, \\
\mathfrak{B}_{\tilde{P}}=\left\{\tilde{w}_{1} \in \mathcal{W}_{e} \mid \tilde{w}_{1}=\left(\tilde{u}_{1}, \tilde{y}_{1}\right) \text { satisfies }(4.18) \text { for some } x\right\}, \\
\dot{x}(t)=f\left(x(t), \tilde{u}_{1}(t-\tau)\right), \quad \tilde{y}_{1}=-x, \quad \tau \in\left(0, \tau_{0}\right] .
\end{gathered}
$$

For any $\tilde{w}_{1-}=\left(\tilde{u}_{1-}, \tilde{y}_{1-}\right) \in \mathcal{W}^{-} \cap \mathfrak{B}_{\tilde{P}}^{-}$, choose $w_{1-}=\left(u_{1-}, y_{1-}\right) \in \mathcal{W}^{-} \cap \mathfrak{B}_{P}^{-}$with $u_{1-}=\tilde{u}_{1-}$ and $y_{1-}(t-\tau)=\tilde{y}_{1-}(t)$ for $t \leq 0$. Then,

$$
\left\|w_{1-}\right\| \leq \max \left\{\left\|\tilde{w}_{1-}\right\|_{(-\infty,-\tau]},\left\|\left(\tilde{u}_{1-}, y_{1-}\right)\right\|_{[-\tau, 0]}\right\} \leq \max \left\{2\left\|\tilde{w}_{1-}\right\|,\left\|y_{1-}\right\|_{[-\tau, 0]}\right\} .
$$

Since $\dot{x}=f(x, u)$ with $f(0,0)=0$ is forward complete, we have by using [20, Lemma 3.5] that $\left\|y_{1-}\right\|_{[-\tau, 0]} \leq \mu(\tau) \nu\left(\left\|w_{1-}\right\|_{(-\infty,-\tau]}+\left\|u_{1-}\right\|_{[-\tau, 0]}\right) \leq \mu\left(\tau_{0}\right) \nu\left(2\left\|\tilde{w}_{1-}\right\|\right)$, and thus we obtain

$$
\left\|w_{1-}\right\| \leq 2\left\|\tilde{w}_{1-}\right\|+\mu\left(\tau_{0}\right) \nu\left(2\left\|\tilde{w}_{1-}\right\|\right),
$$

where $\mu$ is a positive-valued continuous nondecreasing function and $\nu \in \mathcal{K}_{\infty}$. 
Define a map $\Phi: \mathcal{G}_{P}^{w_{1-}} \rightarrow \mathcal{G}_{\tilde{P}}^{\tilde{w}_{1-}}$ by

$$
w_{1+} \triangleq\left(u_{1+}, y_{1+}\right) \mapsto \Phi\left(w_{1+}\right)=\tilde{w}_{1+} \triangleq\left(\tilde{u}_{1+}, \tilde{y}_{1+}\right)=\left(u_{1+}, \tilde{y}_{1+}\right),
$$

and thus $\tilde{y}_{1+}(t)=\left(y_{1-} \wedge y_{1+}\right)(t-\tau) \forall t \geq 0$.

For any $t>h \geq 0$, we have that

$$
\sup \left\{\left|\dot{y}_{1+}(s)\right|: s \in[h, t]\right\} \leq \sup \left\{\left|f\left(-y_{1+}(s), u_{1+}(s)\right)\right|: s \in[h, t]\right\} \leq \rho_{2}\left(\left\|w_{1+}\right\|_{[h, t]}\right) ;
$$

if $t-\tau \geq h$, then

$$
\left|\left(\tilde{y}_{1+}-y_{1+}\right)(t)\right|=\left|y_{1+}(t-\tau)-y_{1+}(t)\right| \leq \tau \cdot \sup \left\{\left|\dot{y}_{1+}(s)\right|: s \in[h, t]\right\},
$$

and if $t-\tau<h$, then

$$
\begin{aligned}
\left|\left(\tilde{y}_{1+}-y_{1+}\right)(t)\right| & \leq\left|\left(y_{1-} \wedge y_{1+}\right)(t-\tau)-y_{1+}(h)\right|+\left|y_{1+}(h)-y_{1+}(t)\right| \\
& \leq 2\left\|w_{1-} \wedge w_{1+}\right\|_{[-\infty, h]}+\tau \cdot \sup \left\{\left|\dot{y}_{1+}(s)\right|: s \in[h, t]\right\} .
\end{aligned}
$$

Hence, for any $t>h \geq 0$ and any $w_{1+} \in \mathcal{G}_{P}^{w_{1-}}$, we have

$$
\left|\left((\Phi-I) w_{1+}\right)(t)\right| \leq \beta_{0}\left(\left\|w_{1-} \wedge w_{1+}\right\|_{(-\infty, h]}, t-h\right)+\tau \cdot \rho_{2}\left(\left\|w_{1+}\right\|_{[h, t]}\right)
$$

with $\beta_{0} \in \mathcal{K} \mathcal{L}$ defined by

$$
\beta_{0}(r, \xi)= \begin{cases}2 r+\frac{r}{1+\xi} & \text { for } r \geq 0, \xi \in[0, \tau) \\ \frac{r}{1+\xi} & \text { for } r \geq 0, \xi \geq \tau\end{cases}
$$

Theorem 3.2 now asserts that, by using (4.14) and (4.20), the perturbed closed-loop system shown in Figure 2 will remain input to output stable if the time delay $\tau$ satisfies

$$
\tau \cdot \rho_{2} \circ(I+\rho) \circ \gamma(s) \leq(I+\varepsilon)^{-1}(s) \quad \forall s \geq 0
$$

for some functions $\rho, \varepsilon$ of class $\mathcal{K}_{\infty}$. In the following, we give a concrete nonlinear example to show that the closed-loop system remains input to output stable under the perturbation of sufficiently small time delay in the plant.

Example 4.3. Consider the feedback configuration in Figure 2. Let $\mathcal{U}=\mathcal{Y}=$ $L^{\infty}(\mathbb{R})$ and $\mathcal{W} \triangleq \mathcal{U} \times \mathcal{Y}$, and let $f(x, u)=\phi(x)+u$ and $\kappa(y)=-k y$, where $k \in \mathbb{R}$ and $\phi: \mathbb{R} \rightarrow \mathbb{R}$ is a memoryless nonlinear function satisfying the sector condition $\phi \in$ Sector $\left(k_{1}, k_{2}\right)$ with $k_{1}, k_{2} \in \mathbb{R}$ and $k_{1} \leq k_{2}<k$, i.e., $\left[\phi(x)-k_{1} x\right]\left[\phi(x)-k_{2} x\right] \leq 0$ for $x \in \mathbb{R}$; this is equivalent to the following statement [5]:

$$
\phi(0)=0 \text { and } k_{1} x^{2} \leq x \phi(x) \leq k_{2} x^{2} \forall x \in \mathbb{R} .
$$

Thus, the nominal closed-loop equations in (4.13) are expressed as

$$
\begin{aligned}
\dot{x} & =-(k x-\phi(x))+u_{0}-k y_{0}, \\
u_{1} & =-k x+u_{0}-k y_{0}, \quad y_{1}=-x .
\end{aligned}
$$

Consider the Lyapunov function candidate $V(x)=x^{2} / 2$, and the derivative of $V$ 
along the trajectories of this system (4.23) is given by

$$
\dot{V}=-x(k x-\phi(x))+x\left(u_{0}-k y_{0}\right) \leq-\left(k-k_{2}\right) x^{2}+x\left(u_{0}-k y_{0}\right),
$$

and thus we get that for any $\varepsilon \in\left(0, k-k_{2}\right)$,

$$
\dot{V} \leq-2 \varepsilon V \quad \forall|x| \geq\left\|u_{0}-k y_{0}\right\| /\left(k-k_{2}-\varepsilon\right) .
$$

Then, by using [16, Theorem 10.4.1], we obtain that for any $\varepsilon \in\left(0, k-k_{2}\right)$, there exists a $\beta_{1} \in \mathcal{K} \mathcal{L}$ such that

$$
|x(t)| \leq \beta_{1}(|x(0)|, t)+\frac{1}{k-k_{2}-\varepsilon}\left\|u_{0}-k y_{0}\right\|_{[0, t)} \quad \forall t \geq 0 .
$$

From (4.23)-(4.24), for any $\varepsilon \in\left(0, k-k_{2}\right)$ we have that (4.14) satisfies with gain function

$$
\gamma(r)=\left(1+k+\frac{1+k}{k-k_{2}-\varepsilon}\right) \cdot r \quad \forall r \geq 0,
$$

where function $\beta \in \mathcal{K} \mathcal{L}$ in (4.14) also depends on $\varepsilon \in\left(0, k-k_{2}\right)$.

Consider again $V(x)=x^{2} / 2$, and the derivative of $V$ along the trajectories of the system $\dot{x}=f(x, u)=\phi(x)+u$ is given by

$$
\dot{V}=x \phi(x)+x u \leq k_{2} x^{2}+\left(x^{2}+u^{2}\right) / 2 \leq\left(2 k_{2}+1\right) V+u^{2} / 2 .
$$

Thus, from [2, Corollary 2.11], we know that the system $\dot{x}=f(x, u)=\phi(x)+u$ is forward complete. Therefore, (4.19) satisfies. Since $|f(x, u)| \leq\left(1+\max \left\{\left|k_{1}\right|,\left|k_{2}\right|\right\}\right)$. $\max \{|x|,|u|\}$, we have that (4.20) satisfies with function $\rho_{2} \in \mathcal{K}_{\infty}$ defined by

$$
\rho_{2}(r)=\left(1+\max \left\{\left|k_{1}\right|,\left|k_{2}\right|\right\}\right) \cdot r \quad \forall r \geq 0 .
$$

From (4.21), (4.25), (4.26), and Theorem 2.19, it follows that the perturbed closedloop system $[\tilde{P}, C]$ will remain input to output stable if for any given $\varepsilon \in\left(0, k-k_{2}\right)$ with time delay $\tau<1 / \omega$, where $\omega \triangleq\left(1+\max \left\{\left|k_{1}\right|,\left|k_{2}\right|\right\}\right)\left(1+k+\frac{1+k}{k-k_{2}-\varepsilon}\right)$.

5. Concluding remarks. By providing a unified construction of an underlying abstract state space applicable to input-output systems defined over a doubly infinite time axis, this paper provides an input-output theory with an integrated treatment of initial conditions, culminating in a statement and proof of a robust stability result. The resulting gap distances take into account both the effect of the perturbation on the state space structure (and hence the initial condition) as well as the input-output response. This complements the robust stability theory of Georgiou and Smith [13] by introducing initial conditions and applies the ideas of the ISS framework in a situation whereby the conventional state-space formalism of ISS is not directly applicable due to variation in the structure of the state space between the nominal and perturbed systems which arise naturally in a robust stability setting.

Acknowledgments. This paper forms essentially a part of the first author's doctoral thesis under the supervision of the second author at the University of Southampton, School of Electronics and Computer Science (ECS). The authors would like to thank the referees for suggestions that have improved this paper. 


\section{REFERENCES}

[1] B. D. O. Anderson and J. B. Moore, Linear Optimal Control, Prentice-Hall, Englewood Cliffs, NJ, 1971.

[2] D. Angeli And E. D. Sontag, Forward completeness, unboundedness observability, and their lyapunov characterizations, Systems Control Lett., 38 (1999), pp. 209-217.

[3] M. A. Arbib And H. P. Zeiger, On the relevance of abstract algebra to control theory, Automatica, 5 (1969), pp. 589-606.

[4] C. Desoer and C.-T. Chen, Controllability and observability of feedback systems, IEEE Trans. Automat. Control, 12 (1967), pp. 474-475.

[5] C. A. Desoer and M. Vidyasagar, Feedback Systems: Input-Output Properties, Academic Press, New York, 1975.

[6] B. A. Francis, A Course in $H_{\infty}$ Control Theory, Springer, New York, 1987.

[7] M. French, Adaptive control and robustness in the gap metric, IEEE Trans. Automat. Control, 53 (2008), pp. 461-478.

[8] M. FRench AND W. BiAn, A biased approach to nonlinear robust stability and performance with applications to adaptive control, SIAM J. Control Optim., 50 (2012), pp. 1220-1243.

[9] M. French, A. Ilchmann, And M. Mueller, Robust stabilization by linear output delay feedback, SIAM J. Control Optim., 48 (2009), pp. 2533-2561.

[10] M. French AND M. Mueller, Nonlinear high gain separation principles and fast sampling results ensuring robust stability, submitted.

[11] J. P. Gauthier and I. Kupka, Deterministic Observation Theory and Applications, Cambridge University Press, Cambridge, 2001.

[12] T. T. Georgiou And M. C. Smith, Biased norms and robustness analysis for nonlinear feedback systems, in Proceedings of the 36th IEEE Conference on Decision and Control, Vol. 1, 1997, pp. 642-643.

[13] T. T. Georgiou And M. C. Smith, Robustness analysis of nonlinear feedback systems: An input-output approach, IEEE Trans. Automat. Control, 42 (1997), pp. 1200-1221.

[14] R. Hermann And A. Krener, Nonlinear controllability and observability, IEEE Trans. Automat. Control, 22 (1977), pp. 728-740.

[15] B. Ingalls, E. D. Sontag, And Y. Wang, Remarks on input to output stability, in Proceedings of the 38th IEEE Conference on Decision and Control, 1999, pp. 1226-1231.

[16] A. Isidori, Nonlinear Control Systems II, Springer, New York, 1999.

[17] Z.-P. Jiang, A. R. Teel, And L. Praly, Small-gain theorem for ISS systems and applications, Math. Control Signals Systems, 7 (1994), pp. 95-120.

[18] T. Kailath, Linear Systems, Prentice-Hall, Englewood Cliffs, NJ, 1980.

[19] R. E. Kalman, P. L. Falb, And M. A. Arbib, Topics in Mathematical System Theory, McGraw-Hill, New York, 1969.

[20] I. KARAFYLLIS, The non-uniform in time small-gain theorem for a wide class of control systems with outputs, Eur. J. Control, 10 (2004), pp. 307-323.

[21] J. LiU, Robust Stability for Nonlinear Control: State-Space and Input-Output Synthesis, Ph.D. thesis, University of Southampton, Southampton, UK, 2014.

[22] A. Nerode, Linear automaton transformations, Proc. Amer. Math. Soc., 9 (1958), pp. 541-544.

[23] J. W. Polderman and J. C. Willems, Introduction to Mathematical Systems Theory: A Behavioral Approach, Springer, New York, 1998.

[24] J. Sakarovitch, Elements of Automata Theory, Cambridge University Press, Cambridge, 2009.

[25] I. W. SANDBerg, On the $L_{2}$-boundedness of solutions of nonlinear functional equations, Bell System Tech. J., 43 (1964), pp. 1581-1599.

[26] I. W. SAndBerg, On the boundedness of solutions of non-linear integral equations, Bell System Tech. J., 44 (1965), pp. 439-453.

[27] E. D. Sontag, Smooth stabilization implies coprime factorization, IEEE Trans. Automat. Control, 34 (1989), pp. 435-443.

[28] E. D. Sontag, Input to state stability: Basic concepts and results, in Nonlinear and Optimal Control Theory, P. Nistri, ed., Springer, New York, 2008, pp. 163-220.

[29] E. D. Sontag AND Y. WANG, On characterizations of the input-to-state stability property, Systems Control Lett., 24 (1995), pp. 351-359.

[30] E. D. Sontag And Y. Wang, New characterizations of input-to-state stability, IEEE Trans. Automat. Control, 41 (1996), pp. 1283-1294.

[31] E. D. Sontag AND Y. WANG, Output-to-state stability and detectability of nonlinear systems, Systems Control Lett., 29 (1997), pp. 279-290.

Copyright (c) by SIAM. Unauthorized reproduction of this article is prohibited. 
[32] E. D. Sontag, Mathematical Control Theory: Deterministic Finite Dimensional Systems, Springer, New York, 1998.

[33] E. D. Sontag And Y. Wang, Notions of input to output stability, Systems Control Lett., 38 (1999), pp. 235-248.

[34] E. D. Sontag And B. Ingalls, A small-gain theorem with applications to input/output systems, incremental stability, detectability, and interconnections, J. Franklin Inst., 339 (2002), pp. 211-229.

[35] M. Vidyasagar, Nonlinear Systems Analysis, 2nd ed., Prentice-Hall, Englewood Cliffs, NJ, 1993.

[36] G. WeIss, Transfer functions of regular linear systems. Part I: Characterizations of regularity, Trans. Amer. Math. Soc., 342 (1994), pp. 827-854.

[37] J. C. Willems, The Analysis of Feedback Systems, MIT Press, Cambridge, MA, 1971.

[38] J. C. Willems, Least squares stationary optimal control and the algebraic Riccati equation, IEEE Trans. Automat. Control, 16 (1971), pp. 621-634.

[39] J. C. Willems, Dissipative dynamical systems-Part I: General theory, Arch. Ration. Mech. Anal., 45 (1972), pp. 321-351.

[40] J. C. Willems, Dissipative dynamical systems - Part II: Linear systems with quadratic supply rates, Arch. Ration. Mech. Anal., 45 (1972), pp. 352-393.

[41] J. C. Willems, Models for Dynamics, Dynamics Reported 2, Springer, New York, 1989, pp. 171-269.

[42] J. C. Willems, Paradigms and puzzles in the theory of dynamical systems, IEEE Trans. Automat. Control, 36 (1991), pp. 259-294.

[43] L. A. Zadeh and C. A. Desoer, Linear System Theory: The State Space Approach, McGrawHill, New York, 1963.

[44] G. Zames, Nonlinear Operators for System Analysis, Technical report 370, MIT Research Laboratory of Electronics, Cambridge, MA, 1960.

[45] G. ZAmes, Functional analysis applied to nonlinear feedback systems, IEEE Trans. Circuit Theory, 10 (1963), pp. 392-404.

[46] G. ZAMES, On the input-output stability of time-varying nonlinear feedback systems-Part I, IEEE Trans. Automat. Control, 11 (1966), pp. 228-238.

[47] G. ZAmEs, On the input-output stability of time-varying nonlinear feedback systems-Part II, IEEE Trans. Automat. Control, 11 (1966), pp. 465-476.

[48] E. ZeIDLer, Nonlinear Functional Analysis and its Applications I: Fixed-Point Theorems, Springer, New York, 1986.

[49] K. Zhou, J. C. Doyle, And K. Glover, Robust and Optimal Control, Prentice-Hall, Englewood Cliffs, NJ, 1995

Copyright (C) by SIAM. Unauthorized reproduction of this article is prohibited. 